This item was submitted to Loughborough's Research Repository by the author.

Items in Figshare are protected by copyright, with all rights reserved, unless otherwise indicated.

\title{
Method to improve catalyst layer model for modelling proton exchange membrane fuel cell
}

PLEASE CITE THE PUBLISHED VERSION

http://dx.doi.org/10.1016/j.jpowsour.2015.04.152

PUBLISHER

(C) Elsevier

VERSION

AM (Accepted Manuscript)

\section{PUBLISHER STATEMENT}

This work is made available according to the conditions of the Creative Commons Attribution-NonCommercialNoDerivatives 4.0 International (CC BY-NC-ND 4.0) licence. Full details of this licence are available at: https://creativecommons.org/licenses/by-nc-nd/4.0/

\section{LICENCE}

CC BY-NC-ND 4.0

\section{REPOSITORY RECORD}

Zhang, Xiaoxian, Yuan Gao, Hossein Ostadi, Kyle Jiang, and Rui Chen. 2015. "Method to Improve Catalyst Layer Model for Modelling Proton Exchange Membrane Fuel Cell”. figshare. https://hdl.handle.net/2134/17950. 


\title{
Method to improve catalyst layer model for modelling proton exchange membrane fuel cell
}

\author{
Xiaoxian Zhang ${ }^{\mathrm{a}}$, Yuan Gao ${ }^{\mathrm{b}}$, Hossein Ostadi ${ }^{\mathrm{c}}$, Kyle Jiang ${ }^{\mathrm{d}}$, Rui Chen ${ }^{\mathrm{e}}$ \\ a School of Engineering, University of Liverpool, Brownlow Street, Liverpool, L69 3GQ UK. \\ e-mail: Xiaoxian.zhang@liverpool.ac.uk \\ ${ }^{\mathrm{b}}$ Clean Energy Automotive Engineering Centre \& School of Automotive Studies, Tongji \\ University, Shanghai 201804, China e-mail: yuangao@tongji.edu.cn \\ b Intelligent Energy, Charnwood Building, Holywell park, Loughborough, Leicestershire, \\ LE113GB, UK. e-mail: Hossein.Ostadi@intelligent-energy.com \\ d School of Mechanical Engineering, University of Birmingham, BirminghamB15 2TT, UK. \\ e-mail: k.jiang@bham.ac.uk \\ e Department of Aeronautical and Automotive Engineering, Loughborough University, \\ Leicestershire LE11 3TU, UK. e-mail: R.Chen@lboro.ac.uk
}




\begin{abstract}
Correctly describing oxygen reduction within the cathode catalyst layer (CL) in modelling proton exchange membrane fuel cell is an important issue remaining unresolved. In this paper we show how to derive an agglomerate model for calculating oxygen reactions by describing dissolved oxygen in the agglomerates using two independent random processes. The first one is the probability that an oxygen molecule, which dissolves in the ionomer film on the agglomerate surface, moves into and then remains in the agglomerates; the second one is the probability of the molecule being consumed in reactions. The first probability depends on CL structure and can be directly calculated; the second one is derived by assuming that the oxygen reduction is first-order kinetic. It is found that the distributions functions of the first process can be fitted to a generalised gamma distribution function, which enables us to derive an analytical agglomerate model. We also expend the model to include oxygen dissolution in the ionomer film, and apply it to simulate cathode electrodes. The results reveal that the resistance to oxygen diffusion in ionomer film and agglomerate in modern CL is minor, and that the main potential loss is due to oxygen dissolution in the ionomer film.
\end{abstract}

Key words: Cathode catalyst layer; Agglomerate model; Oxygen dissolution; Ionomer film; Cathode electrode; Pore-scale modelling 


\begin{tabular}{|c|c|}
\hline \multicolumn{2}{|c|}{ Nomenclature } \\
\hline$A_{1}$ & Specific outer surface area of ionomer film \\
\hline$c^{\prime}$ & Concentration of dissolved oxygen within agglomerates \\
\hline$C_{i}$ & Average molar concentration of gas i in inter-agglomerate pores \\
\hline$C_{o}$ & Concentration of dissolved oxygen in outer surface of ionomer film \\
\hline C & Total molar concentration of all gases in inter-agglomerate pores \\
\hline \multirow{2}{*}{$D_{o}^{e q}$} & Concentration of dissolved oxygen in equilibrium with gaseous \\
\hline & oxygen in inter-agglomerate pores \\
\hline $\mathrm{C}_{\mathrm{ref}}$ & Reference dissolved oxygen concentration \\
\hline $\mathrm{D}_{0}$ & Diffusion coefficient of dissolved oxygen in ionomer \\
\hline$D^{\prime}$ & Effective diffusion coefficient of dissolved oxygen in agglomerates \\
\hline$D_{i j}^{e q}$ & Effective binary diffusion coefficient of gases $i$ and $j$ in CL and GDL. \\
\hline$D_{i j}$ & Binary diffusion coefficient of gases $i$ and $j$ in free space \\
\hline$E_{r}^{\prime}$ & Effectiveness factor of agglomerate model \\
\hline $\mathrm{E}_{\mathrm{th}}$ & Theoretical oxygen reduction potential \\
\hline $\mathrm{E}$ & Cell voltage \\
\hline$f_{\text {in }}(\varphi)$ & Tortuosity of GDL in in-plane direction \\
\hline$f_{\text {throu }}(\varphi)$ & Tortuosity of GDL in through-plane direction \\
\hline $\mathrm{F}$ & Faraday constant \\
\hline $\mathrm{H}_{\mathrm{O} 2}$ & Henry’s constant for oxygen dissolution in ionomer \\
\hline$I$ & Average current density \\
\hline $\boldsymbol{i}$ & Local current density \\
\hline $\mathrm{i}_{\text {ref }}$ & Reference exchange current density \\
\hline $\mathrm{k}_{\mathrm{c}}$ & Average oxygen reduction rate coefficient in agglomerate \\
\hline $\mathrm{k}_{\mathrm{d}}$ & Gaseous oxygen dissolution rate coefficient in ionomer film \\
\hline$N_{i}$ & Molar flux of gas $i$ in inter-agglomerate pores \\
\hline po & Partial pressure of gaseous oxygen in inter-agglomerate pores \\
\hline $\mathrm{R}$ & Gas constant \\
\hline $\mathrm{R}_{\mathrm{m}}$ & Resistance of the membrane \\
\hline $\mathrm{R}_{0}(t)$ & Mass of dissolved oxygen in agglomerate at time $t$ \\
\hline $\mathrm{R}_{\mathrm{e}}(t)$ & Average oxygen reduction rate in the catalyst layer at time $t$ \\
\hline $\mathrm{S}_{\mathrm{a}}$ & Volumetric reactive surface area of catalyst in agglomerate \\
\hline
\end{tabular}




$\begin{array}{cl}\mathrm{T} & \text { Temperature } \\ \mathrm{V}_{\mathrm{i}} & \text { Volume of voxel in the 3D image of the reconstructed catalyst layer } \\ \mathrm{v}_{\mathrm{i}} & \text { Average volume of the ionomer in each agglomerate voxel } \\ \alpha_{\mathrm{c}} & \text { Cathode transfer coefficient } \\ \beta & \text { Agglomerate model parameter } \\ \alpha & \text { Agglomerate model parameter } \\ \mathrm{k} & \text { Agglomerate model parameter } \\ \eta & \text { Local overpotential } \\ \varphi & \text { GDL porosity } \\ \phi_{\mathrm{s}} & \text { Solid potential } \\ \phi_{\mathrm{m}} & \text { Electrolyte potential } \\ \sigma_{\mathrm{m}} & \text { Protonic conductivity of CL } \\ \sigma_{\mathrm{s}} & \text { Electronic conductivity of GDL and CL } \\ \theta & \text { Intra-agglomerate porosity } \\ \varepsilon & \text { Size of the voxels in the 3D CL images } \\ \lambda & \text { Average thickness of the ionomer film }\end{array}$




\section{Introduction}

Species transport and electrochemical reactions in the gas-diffusion layer (GDL) and catalyst layer (CL) within the polymer electrolyte membrane (PEM) fuel cell occur at pore scale. In practical fuel cell modelling, however, the geometries of these pores cannot be explicitly resolved, and their impact on different fuel cell processes is instead described by volumetric average parameters, such as effective diffusion coefficients for species diffusion and effective conductivities for charge transport [1]. How to accurately describe these porescale processes in a fuel cell model is crucial, and the most challenging one is the description of oxygen diffusion and reaction in the cathode CL [2, 3].

Most catalyst layers in PEM fuel cells use carbon grains to support the catalyst, and the carbon grains are further bound by an ionomer $[4,5]$. During manufacturing, the carbon grains tend to aggregate, forming porous agglomerates [6] with their outer surfaces coated by a thin ionomer film [7, 8]. As such, the catalyst layer has a bi-mode pore structure in which the pores inside the agglomerates (intra-agglomerate pores) are much smaller than the pores between the agglomerates (inter-agglomerate pores). The ionomer film provides pathway for protons to transport, whilst the inter-agglomerate pores provide pathways for gaseous species to move [9].

In the cathode CL, gaseous oxygen moves from the GDL into the inter-agglomerate pores first, and then dissolves in the liquids before moving into the catalyst sites within the agglomerate to electrochemically react with protons and electrons[10]. In fuel cell modelling, the movement of gaseous oxygen in the inter-agglomerate pores is often assumed to be diffusive and modelled by a volumetric average diffusion equation. The diffusion and reaction of the dissolved oxygen within the agglomerate, however, cannot be explicitly resolved. Instead, they are treated as a sink term and calculated by a method known as agglomerate model in the literature [11]. 
The oxygen needs to overcome a number of resistances, including its dissolution in the ionomer film and the consequent diffusion in the ionomer film and the agglomerate [12], before it can react with electron and proton on the catalyst sites. The relative significance of each resistance, however, is poorly understood. With a few exceptions [13-15], the most common method used in the literature to describe these resistances is the spherical agglomerate model by idealising the CL as a packing of isolated spheres [16, 17]. This idealisation not only oversimplifies the CL structure [4, 18-21], but is also self-defeating. For example, its assumption of isolated and non-touched spheres physically makes the CL insulate to electrons as electrons can only transport through the carbon grains. Hence, the spherical agglomerate model is just to introduce a resistance to oxygen diffusion rather than a geometrical description of the CL structure. This explains why the agglomerate diameter used in the spherical agglomerate model varies so widely in the literature, ranging from 200nm to $6000 \mathrm{~nm}$, far bigger than the average agglomerate size revealed by tomography, which is approximately 100nm [22, 23]. The thickness of the ionomer film used in the spherical models is up to $80 \mathrm{~nm}$, also much thicker than real ionomer film, which is only about $10 \mathrm{~nm}$ thick [5]. The inferiority of the spherical model is well known, but still remains the dominant models in the literature due to the lack of alternatives [3, 24-28].

There has been an increase over the past few years in use of nano-tomography to characterise CL at resolutions of a few nanometres [4, 18-20, 29]. This makes simulation of oxygen diffusion and reduction in a real CL feasible, and can be used to directly calculate the electrochemical reaction rate without need to simplify the CL structure [30, 31]. The concern over this direct method is its computational cost. In fuel cell modelling, the CL is often divided into a number of grids. Since the reaction rate depends on overpotential, which varies spatially in the CL, the above direct method needs to calculate the reaction rate in each numerical grid using pore-scale simulation; this is computationally expensive. For practical 
use, therefore, it is ideal to derive a simple formula that can adequately describe the average reaction rate by explicitly taking all agglomerate processes into account. We made such an effort by assuming that the ionomer film was thin [32]. In this paper, we show how to derive the agglomerate model for a given agglomerate structure by describing oxygen movement and reaction within it as two independent random processes. We also apply the model to simulate a cathode electrode in an attempt to elucidate the relative significance of each resistance to oxygen diffusion in the agglomerates.

\section{Background and electrode model}

Species transport and reaction in the GDL and CL take place at pore scale and depend on their pore geometries. In practical fuel cell modelling, the impact of these pore geometries is described using effective parameters by averaging the individual pores out. For the cathode electrode shown in Fig.1, gas transport in the inter-agglomerate pores within the CL and all pores in the GDL is described by volumetric average diffusion equations with the GDL and the CL distinguished by using different effective diffusion coefficients. However, the oxygen diffusion and reduction inside the agglomerate cannot be explicitly resolved, and they are instead included in the average diffusion equations as a sink term represented by an agglomerate model. In this paper, we consider only water vapour, nitrogen and oxygen in the cathode electrode. The transport of the three gases in both GDL and CL is described by the following equations [26]:

$$
\begin{aligned}
& \nabla \cdot N_{i}=R_{e i}, \\
& \nabla C_{i}=\sum_{i} \frac{C_{i} C_{j}}{C^{2} D_{i j}^{e f f}}\left(\frac{N_{j}}{C_{j}}-\frac{N_{i}}{C_{i}}\right),
\end{aligned}
$$

where $C_{i}$ is the volumetric average molar concentration of gas $i, D_{i j}^{e f f}$ is the effective binary diffusion coefficient of gases $i$ and $j, C$ is total molar concentration, $N_{i}$ is the volumetric 
average molar flux of gas $i$, and $R_{e i}$ is the average reaction rate of gas $i$. Since electrochemical reaction takes place only in the CL, $R_{e i}$ is zero in the GDL.

For oxygen, its volumetric average reduction rate in the CL is often described by the following agglomerate model[17]:

$$
R_{e o}=k_{c} E_{r} \frac{R T p_{O}}{H_{O_{2}}}
$$

where $E_{r}$ is an effectiveness factor describing the decrease of reduction rate due to a combined impact of catalyst loading, operating conditions, oxygen dissolution in the ionomer film and its consequent diffusion in the ionomer film and agglomerate; $R$ is the gas constant; $T$ is temperature; $p_{O}$ is the partial pressure of the gaseous oxygen in the inter-agglomerate pores; $H_{\mathrm{O} 2}$ is the Henry's constant for oxygen dissolution in the liquid; and $k_{\mathrm{c}}$ is the reduction rate coefficient that describes the impact of protonic and electronic potentials on electrochemical reaction; it is often described by the following Butler-Volmer kinetics $k_{c}=\frac{S_{a} i_{r e f}}{4 F c_{\text {ref }}}\left[\exp \left(\frac{\alpha_{c} \eta F}{R T}\right)-\exp \left(\frac{\left(\alpha_{c}-1\right) \eta F}{R T}\right)\right]$,

where $S_{a}$ is the electrochemically active surface area of the catalyst in a unit volume of agglomerate, $F$ is the Faraday constant, $i_{\text {ref }}$ is reference exchange current density, $c_{\text {ref }}$ is reference oxygen concentration, $\alpha_{c}$ is cathode transfer coefficient and $\eta$ is volumetric average local overpotential.

Proton transport is restricted to the CL and the membrane, and described by the following volumetric-average equation:

$$
\begin{aligned}
& \nabla \cdot \boldsymbol{i}=4 F \cdot R_{e o}, \\
& \boldsymbol{i}=-\sigma_{m} \nabla \phi_{m},
\end{aligned}
$$

where $\boldsymbol{i}$ is volumetric average current density, $\sigma_{\mathrm{m}}$ is effective protonic conductivity of the CL, and $\phi_{m}$ is volumetric-average potential of the ionomer. 
Electron transport is through the solids in both GDL and CL, and described by the following volumetric average equation:

$$
\begin{aligned}
& \nabla \cdot \boldsymbol{i}=-4 F \cdot R_{e o}, \\
& \boldsymbol{i}=-\sigma_{s} \nabla \phi_{s},
\end{aligned}
$$

where $\sigma_{\mathrm{s}}$ is effective electronic conductivity of the GDL or the CL, and $\phi_{s}$ is the volumetric average potential of the carbon fibres or carbon grains in the GDL and the CL, respectively.

The above equations have to be solved numerically. In numerical solution, the cathode electrode shown Fig.1 is divided into a number of grids [33]. Species concentrations and potentials of the solid and electrolyte are assumed to be constants in each grid. As an illustration, we take the CL grid shown in Fig. 1 as an example, the agglomerates in which are not explicitly modelled but implicitly described in the above equations by $R_{e o}$. The value of $R_{e o}$ in the gird depends not only on its average gaseous oxygen concentration and protonic and electronic potentials as shown in Eq.(2), but also on the properties of the CL, including its catalyst loading, ionomer films, the ability of the agglomerate to conduct oxygen, and oxygen dissolution and its consequent diffusion in the ionomer film and the agglomerate. One challenge in fuel cell modelling is how to accurately represent these non-resolved properties and processes in the agglomerate model.

\section{The agglomerate model}

The agglomerate model is to calculate the average electrochemical reaction rate within the numerical CL grid shown in Fig.1 using the average gaseous oxygen concentration and protonic and the electronic potentials, with other non-resolved processes and factors implicitly described by the effectiveness factor $E_{r}$.

\subsection{Statistical analysis of dissolved oxygen in the agglomerate}


Referring to Fig. 1, when the gaseous oxygen moves into the inter-agglomerate pores from the GDL, it needs to dissolve in the ionomer film first before it can diffuse into the catalyst sites inside the agglomerate to electrochemically react with protons and electrons. The diffusion and reaction of each dissolved oxygen molecule in the agglomerate can be described as two independent random processes. The first one is the probability that an oxygen molecule, which dissolves in the ionomer film at time $t=0$, moves into and then remains in the agglomerate, excluding the ionomer film, at time $t$ under condition that there is no electrochemical reaction. Therefore, this probability depends only on agglomerate geometry and the ability of the agglomerate to conduct oxygen; it is independent of operation conditions. We use $f(t)$ to describe this probability. The second process is for electrochemical reaction; it is the probability that the above molecule will be consumed in an electrochemical reaction at time $t$; we represent this probability by $\bar{f}(t)$.

If there are $C$ ' oxygen molecules that dissolve in the ionomer film surface at time $t=0$ within the CL grid shown in Fig.1, we use $f\left(t, x_{i}\right)$ to describe the probability of one of these molecules, which is located at $x_{i}$ on the ionomer film surface at $t=0$ and will remain in the agglomerate at time $t$. Among the $C$ 'molecules, the number of molecules that are still in the agglomerate at time $t$ is hence

$$
R_{0}(t)=C^{\prime} \sum_{i=1}^{C^{\prime}} f\left(t, x_{i}\right)
$$

The average probability that one of those molecules remains in the agglomerate at time $t$ is $g(t)=\sum_{i=1}^{C^{\prime}} f\left(t, x_{i}\right) / C^{\prime}$

The oxygen reduction in the CL is assumed to be one-order kinetic; that is, the probability of a molecule being consumed in a reduction reaction within a unit time period is $k_{c}$. Therefore, the probability that a molecule, which enters the agglomerate at $t=0$ and is still alive at time $t$, is $\bar{f}(t)=\exp \left(-k_{c} t\right)$. Among the $C^{\prime}$ molecules that enter the agglomerate after 
dissolving in the ionomer film surface at $t=0$, those still in the agglomerate at time $t$ in the CL grid shown in Fig.1 are

$$
R_{0}(t)=C^{\prime} \cdot g(t) \exp \left(-k_{c} t\right)
$$

Since oxygen reduction is assumed to be first-order kinetic, the rate at which the number of molecules is consumed by electrochemical reactions at time $t$ is

$$
R_{e}(t)=k_{c} \cdot C^{\prime} \cdot g(t) \exp \left(-k_{c} t\right)
$$

If oxygen continuously flows into the grid shown in Fig. 1 such that the number of dissolved oxygen molecules in the ionomer film surface at time $t$ is kept at $C_{0}(t)$, the number of molecules remaining inside the agglomerate at time $t$ is

$$
R_{0}(t)=\int_{0}^{t} C_{0}(\tau) g(t-\tau) \exp \left[-k_{c}(t-\tau)\right] d \tau
$$

The rate at which the number of molecules is consumed in the reduction reactions at time $t$ is

$$
R_{e}(t)=k_{c} \int_{0}^{t} C_{0}(\tau) g(t-\tau) \exp \left[-k_{c}(t-\tau)\right] d \tau
$$

Eq.(11) is a general agglomerate model, linking the average reduction rate to CL structure through the probability distribution function $g(t)$. Once $g(t)$ is known, Eq.(11) can be analytically or numerically solved to calculate the average reaction rate at time $t$. When $t \rightarrow \infty$, the cell reaches steady state and the reaction rate calculated from Eq.(11) is the rate defined in Eq.(2). It was found in our previous work that $g(t)$ can be approximated by the gamma distribution function when the ionomer film is thin [32]. As the ionomer film increases, however, the gamma distribution is unable to describe $g(t)$.

\section{2 . Link agglomerate model to catalyst layer structure}

The impact of CL structure on electrochemical reaction is described by the distribution function $g(t)$. To explain how to calculate this distribution function for a given CL with its agglomerate coated by ionomer film, we used the CL shown in Fig. 2A as an example. It is a 
cathode CL acquired using FIB/SEM tomography at resolution of 5 nanometres. The FIB/SEM is unable to distinguish carbon grains and ionomer, and we hence numerically coated ionomer films over the agglomerate. Fig. 2B shows a CL grid we simulated with its agglomerate numerically coated by an ionomer film. To investigate the impact of ionomer content, we coated the agglomerate by ionomer films with different thicknesses. Fig. 3 shows the sections of the CL with its agglomerate coated by ionomer film with dimensionless thickness of $\lambda^{\prime}=\lambda / \varepsilon=0,1,2,3$ respectively, where $\lambda$ is the average thickness of the ionomer film and $\varepsilon$ is the side length of the voxels in the acquired image.

The probability distribution function $g(t)$ depends on the agglomerate structure and ionomer film. To calculate it, we tracked the movement of oxygen within the agglomerates by setting the dissolved oxygen concentration on the outer surface of the ionomer film to be a constant and the electrochemical reaction rate to be zero. That is, set $C_{0}(t)=C_{1}$ and $k_{c}=0$ in Eq. (10), meaning that the concentration of dissolved oxygen on the outer surface of the ionomer surface, i.e., the interface between the red and the blue in Fig.2B and Fig.3, is $C_{1}$. Under these conditions, Eq.(10) reduces to

$$
R_{0}(t)=C_{1} \int_{0}^{t} g(\tau) d \tau
$$

from which we have

$$
g(t)=\frac{1}{C_{1}} \frac{d R_{0}(t)}{d t}
$$

where $R_{0}(t)$ is the mass of oxygen in the agglomerate at timet .

Since the diffusion of oxygen from the inter-agglomerate pores into the agglomerates shown in Fig.2 is controlled by the agglomerate geometry, the increase of $R_{0}(t)$ with time also depends on the agglomerate structure. This naturally links the agglomerate structure to agglomerate model through $g(t)$. We simulated the diffusion of the dissolved oxygen from 
inter-agglomerates pores into the agglomerate shown in Fig. 2B using pore-scale simulations. The pore-scale simulation is based on the finite volume method by taking each voxel in the FIB/SEM image as an element at which a discrete mass-balance equation with the concentration of dissolved oxygen in all agglomerate and ionomer voxels as the variables. The oxygen concentration on the outer surface of the ionomer film, i.e., the interface between the red and blue in Fig.2B, was kept at $\mathrm{C}_{1}$. The discrete linear system of equations was solved by the preconditioned conjugate-gradient iterative method[34]. The diffusion coefficients of the oxygen in the ionomer film and the agglomerates are given in Table 1 . The size of the CL in Fig.2B is $1 \mathrm{um}^{3}$, comparable to the CL grid shown in Fig.1. During the simulations, the number of molecules in the agglomerate, excluding those in the ionomer film, was counted, from which the probability distribution function $g(t)$ was calculated using Eq.(13). Fig. 4 shows the change of $g(t)$ with time for the CL grid shown in Fig. 2B when its agglomerate is coated by ionomer films of different thicknesses. It is evident that without ionomer film, $g(t)$ peaks at $t=0$, whilst as the ionomer film thickness increases, the time at which $g(t)$ peaks shifts away from $t=0$.

The distribution functions shown in Fig.4 can be directly used to calculate the oxygen reduction reaction rate by numerically integrating Eq. (11) by setting $t \rightarrow \infty$. However, since the reduction rate coefficient $k_{c}$ is a function of the local overpotential which changes spatially in the CL, numerically integrating Eq.(11) for all numerical grids is computationally expensive. Therefore, it is practically useful to find a formula that can describe all these distribution functions such that, after being inserted to Eq.(11), it could lead to a simple analytical formula to calculate the average reaction rate.

Unfortunately, the number of probability distribution functions with analytical solutions is limited in the literature, and none of them is able to fit all distribution functions shown in Fig. 4. We therefore, following Cvetkovic [35], generalise the gamma distribution function. 
The generalised gamma distribution function cannot be analytically expressed by $t$, and is defined by its Laplace transform as follows:

$$
G(s)=\int_{0}^{\infty} g(t) e^{-s t} d t=\left[1+(s / \alpha)^{\beta}\right]^{-k},
$$

where $\alpha, \beta$ and $k$ are parameters. The distribution function defined by Eq.(14) reduces to the gamma distribution when $\beta=1$, and to the exponential distribution when $\beta=k=1$. As the function $g(t)$ is calculated by tracking the movement of oxygen within the agglomerate and the ionomer film without any reactions, the three model parameters depend only on agglomerate geometry, including the ionomer film, and their ability to diffuse the oxygen.

To estimate the three model parameters defined in Eq. (14) for the agglomerate coated by different ionomer films, we first Laplace-transformed the calculated probability distribution functions $g(t)$ shown in Fig. 4 and then fitted them to Eq. (14). The comparison is shown in Fig. 5 with the associated fitting parameters.

The agglomerate model used in the literature is to calculate the volumetric average electrochemical reaction rate in a CL grid as shown in Fig.1 when the system is in steady state. This is equivalent to Eq. (11) by letting $t \rightarrow \infty$, that is,

$$
R_{e o}=\lim _{t \rightarrow \infty} k_{c} \int_{0}^{t} C_{o}(\tau) g(t-\tau) \exp \left[-k_{c}(t-\tau)\right] d \tau
$$

As proven in Appendix A, we can analytically derive the following agglomerate model by replaying $g(t)$ in Eq.(15) with that defined in Eq.(14):

$$
\begin{aligned}
& R_{e o}=E_{r} \cdot k_{c} C_{0} \\
& E_{r}=\left[1+\left(k_{c} / \alpha\right)^{\beta}\right]^{-k},
\end{aligned}
$$

where $E_{r}$ is the effectiveness factor, and $C_{0}$ is the dissolved oxygen concentration in the outer surface of the ionomer film. From the above discussion, it is understood that the effect of diffusion coefficient of the agglomerate, agglomerate geometry and the ionomer film on the 
oxygen reduction rate $R_{e o}$ is described by the three CL structure parameters $\alpha, \beta$ and $k$, respectively, and the impact of catalyst loading is described by $k_{c}$ as shown in Eq.(3). The three CL structure parameters are independent of $k_{c}$ and the catalyst loading.

\subsection{Agglomerate model including oxygen dissolution}

Most agglomerate models used in the literature assumed the oxygen dissolution in the ionomer film is fast and not a limiting factor. Whilst this approximation might be rationale at low potential, experiments recently showed that it could give rise to significant errors at high overpotential [36].

The dissolving rate of oxygen in ionomer film depends on the concentration of the dissolved oxygen in the outer surface of the ionomer film and the gaseous oxygen concentration in the inter-agglomerate pores. It can be described by the following first-order kinetic [36]:

$J=k_{d}^{o}\left(C^{e q}-C_{0}\right)$

where $k_{d}^{o}$ is the dissolving rate coefficient, and $C^{\mathrm{eq}}$ is the dissolved oxygen concentration that is in equilibrium with the gaseous oxygen in the inter-agglomerate pores. If the partial pressure of the gaseous oxygen in the inter-agglomerate pores is $p_{o}, C^{\mathrm{eq}}$ can be estimated from the Henry's law, $C^{e q}=H_{O 2} p_{o}$. In the CL grid shown in Fig.2B, if the contact area between the gaseous oxygen and the ionomer film is $S$, the rate at which the gaseous oxygen dissolves into the ionomer film is

$J=k_{d}^{o} \oiint_{S}\left(C^{e q}-C_{0}\right) \cdot d s$.

If the change of $C_{0}$ over $S$ is small and can be approximated by a constant, we have

$$
J \approx k_{d}^{o} S_{1}\left(C_{o}^{e q}-C_{0}\right)
$$

where $S_{1}$ is the outer surface area of the ionomer film. At steady state, $J$ is equal to the electrochemical reaction rate within the grid, and from mass balance we hence have 


$$
S_{1} \cdot k_{d}^{o}\left(C_{o}^{e q}-C_{0}\right)=V \cdot E_{r} \cdot k_{c} \cdot C_{0},
$$

where $V$ is the volume of the agglomerate in the CL grid. Solving for $C_{0}$ yields

$$
\begin{aligned}
& R_{e o}=E_{r}^{\prime} k_{c} C^{e q}, \\
& E_{r}^{\prime}=\left(\frac{1}{E_{r}}+\frac{k_{c}}{k_{d}^{o} A_{1}}\right)^{-1},
\end{aligned}
$$

where $A_{1}=S_{1} / V$ is the specific outer surface area of the ionomer film in the CL. If oxygen dissolution is fast in comparison to the electrochemical reaction rate such that $k_{c} / k_{d}^{o} A_{1}<<1$, the limitation of oxygen dissolution is negligible and Eq.(21) reduces to the original agglomerate model of Eq.(16).

\subsection{Test the agglomerate model}

To test the accuracy of the electrochemical reaction rate predicted by Eq. (21) using the parameters directly estimated from the CL structure, we simulated oxygen diffusion and reaction in the agglomerate shown in Fig. 2B using pore-scale simulation by assuming that the movement of dissolved oxygen in the agglomerate is diffusive. In the pore-scale simulation, the diffusion and electrochemical reaction of the dissolved oxygen in the agglomerate shown in Fig.2B were described by the following equation:

$$
\begin{aligned}
& \nabla \cdot D \nabla c^{\prime}-k_{1} c^{\prime}=0, \\
& \left.D \nabla c^{\prime}\right|_{\Omega}=k_{d}\left(C_{o}^{e q}-c^{\prime}\right) \\
& k_{c}=k_{0}\left[\exp \left(\frac{\alpha_{c} \eta F}{R T}\right)-\exp \left(\frac{\left(\alpha_{c}-1\right) \eta F}{R T}\right)\right] \\
& k_{0}=\frac{S_{a} i_{\text {ref }}}{4 F C_{\text {ref }}} \\
& \text { within the ionomer film: } D=D_{0}, \quad k_{1}=0 \\
& \text { in the agglomerates: } \quad D=D^{\prime}, \quad k_{1}=k_{c}
\end{aligned}
$$

where $c$ ' is the concentration of the dissolved oxygen in the ionomer within the agglomerate, and $\Omega$ is the interface between the inter-agglomerate pores and the outer surface of the ionomer film, that is, the interface between the red and the blue shown in Figs. 2 and 3; $D_{0}$ 
and $D^{\prime}$ are the diffusion coefficients of the dissolved oxygen in the ionomer film and the agglomerate, respectively.

In each simulation, the average electrochemical reaction rate in the CL grid was calculated from

$$
R_{e o}=\frac{\sum_{i=1}^{M} v_{i} k_{c} c_{i}{ }^{\prime}}{\sum_{i=1}^{M} V_{i}},
$$

where $v_{\mathrm{i}}$ is the volume of the ionomer in ith voxel, and $V_{\mathrm{i}}$ is the volume of ith voxel, and $M$ is the total number of the agglomerate voxels in the image.

We express the reaction rate calculated from Eq.(23) as follows :

$$
R_{e o}=E_{r} k_{c} C^{e q}
$$

and we can therefore calculate the effectiveness factor in Eq.(24) from the pore-scale simulations as follows

$$
E_{r}=\frac{\sum_{i=1}^{M} V_{i} c_{i}{ }^{\prime}}{\sum_{i=1}^{M} V_{i}} .
$$

The effectiveness factors calculated from Eq.(25) is compared with that predicted by Eq.(21) using the parameters estimated from the CL structure shown in Fig. 5.

\section{Electrode modelling}

A numerical model for the PEM fuel cell was developed in attempts to prove that the proposed model accurately describes the impact of agglomerate geometry and the ionomer films on electrochemical reaction. The model is two dimensional and based on the method presented in [17], which has been widely used in the literature to model cathode electrode. For oxygen reduction, we used the kinetic parameters given in [17], except stated otherwise, whilst for gas diffusion in GDL and CL, we used the results published recently in the literature[1, 37]. The GDL is anisotropic for both gas diffusion and electronic conduction, and its effective diffusion coefficient and electronic conductivity in both in-plane and 
through-plane directions were taken from our previous work [1]. We also calculated the effective diffusion coefficient of the CL with both bulk and Knudsen diffusions in consideration using the method developed in our previous work[37].

Proton transport in the CL is mainly through the ionomer. An increase in ionomer film thickness is likely to enhance proton conductivity, but decrease the gas diffusion coefficients. In this paper, however, we assumed this impact is minor and used the same diffusion coefficients and protonic conductivity in all simulations. The model parameters used in all simulations are given in Table 1.

Fig. 1 shows the simulated electrode. Because of symmetry, we only simulated part of it as shown in Fig.1. We take the oxygen electrode as reference electrode, and the solid potential at the interface between the GDL and the land is zero as a result. Hence, the local overpotential is the difference between the solid potential and the electrolyte potential. We set the electrolyte potential at the CL-membrane interface from $0 \mathrm{~V}$ to $1.2 \mathrm{~V}$ to simulate the electrode, and its difference from the solid potential at the GLD-land interface is cathode potential. The average current density in the electrode was calculated from

$$
I=\frac{1}{A} \iiint_{\Lambda} \nabla \cdot \mathbf{i} d \Lambda
$$

where $\Lambda$ is the simulated CL domain enclosed by the broken lines shown in Fig.1, and A is the nominal area of the CL in the simulated domain.

The two vertical boundaries of the domain were treated as no-flux boundaries for all species and charges. The CL-membrane interface was a specified potential boundary for proton transport, and no-flux boundary for other species and electron. The CL-GDL interface was not explicitly treated as a boundary, but implicitly solved by assigning different diffusion coefficients and conductivities to GDL and CL. The GDL-channel interface was solved as a specified concentration boundary for gases, and no-flux boundary for charges. In contrast, the GDL-land interface was solved as a specified potential boundary for electron, and no-flux 
boundary for proton and other species. The domain was divided into a number of equal grids and the size of each grid in the in-plane and through plane directions was $3 \mu \mathrm{m}$ and $2 \mu \mathrm{m}$ respectively. For the CL, this is comparable to the CL grid we simulated previously for calculating the agglomerate model parameters.

\section{Results and discussion}

\subsection{Agglomerate model parameters}

In fitting the simulated distribution functions to Eq. (14), it was found that we can fit all them using the same $\alpha$ and $\beta$ by changing the parameter $k$ only to describe the impact of ionomer films. Except stated otherwise, in what follows the time $t$ is normalised by $t^{\prime}=t D^{\prime} / \varepsilon^{2}$ and length $x$ by $x^{\prime}=x / \varepsilon$. The parameters $\beta$ and $k$ have no unit and their values do not change after the normalisations. The comparison between the best-fitting of Eq.(14) and the Laplace-transformed distribution functions calculated from the CL structure in Fig.2B is shown in Fig. 5; they agree well. The values of the normalised three parameters $\alpha^{\prime}$ and $\beta$ are 0.13 and 0.79 respectively, and the value of $k$ increases from 1.0, when there was no ionomer film, to 1.71 , after the normalised ionomer-film thickness increased to $\lambda^{\prime}=3$. The increase of $k\left(\lambda\right.$ ') with the normalised ionomer-film thickness $\lambda^{\prime}$ can be fitted to $k^{\prime}\left(\lambda^{\prime}\right)=k\left(\lambda^{\prime}\right)-k(0)=0.099 \lambda^{1.8}$ and the goodness of the fitting is shown in Fig. 6A.

The gamma distribution function peaks at $t=0$ when $k \leq 1$ and at $t=t_{1}>0$ when $k>1$.

The generalized gamma distribution function does not have an analytical solution in time, but the role of the parameter $k$ in it is similar to that in the gamma distribution. In practical application, we can safely assume $k=1$ for agglomerate without ionomer film and $k>1$ for agglomerate with ionomer films. The increase of $k$ with ionomer film thickness can be estimated from the relationship shown in Fig. 6A. 
The parameter $\alpha$ has a unit of $s^{-1}$ and depends on the agglomerate geometry and diffusion coefficient of the dissolved oxygen within it. The effective diffusion coefficient of oxygen in agglomerate increases with temperature [38]:

$$
D^{\prime}=\left(1.427 \times 10^{-2} T-4.2185\right) 10^{-9} \theta^{1.5}\left(\mathrm{~m}^{2} \mathrm{~s}^{-1}\right) .
$$

The resolution of the image shown in Figs. 2 and 3 is 5 nanometres. Therefore, the value of the parameter $\alpha$ increases with temperature and the increase is shown in Fig. 6B.

\subsection{Accuracy of the agglomerate model}

The agglomerate model in Eq. (21) predicts the decrease of the electrochemical reaction rate due to oxygen diffusion and dissolution in the ionomer film and agglomerate, using the parameters directly estimated from the CL structures. Fig. 7 shows its accuracy in comparison with the effectiveness factors directly calculated from the pore-scale simulations of oxygen diffusion and reaction in the CL grid shown in Fig. 2B when the oxygen dissolution is not a limiting factor. Overall, they agree well, although there is a slight discrepancy because the generalised gamma distribution function is an approximation rather than an exact description of the probability distribution functions.

We also tested the accuracy of Eq.(21) when oxygen dissolution becomes a limiting factor. In the simulations, the dissolution rate coefficient is normalised by $k_{d}=k_{d}^{o} \varepsilon / D_{0}$. We take the CL with its agglomerate coated with ionomer film of average thickness of $\lambda^{\prime}=3$ as an example to demonstrate the accuracy of Eq.(21). Fig. 8 compares the effectiveness factors directly calculated from pore-scale simulations of oxygen diffusion and reactions under two contrasting dissolving rate coefficients with that predicted by Eq.(21). The low dissolving rate coefficient of $k_{d}=0.01$ is taken from Shah et al.[22]. Overall, the comparisons show a good agreement. There is a slight difference because the generalised gamma distribution 
function is an approximation of the real distribution functions and the dissolved oxygen concentration over the outer surface of the ionomer film was assumed to be a constant.

\subsection{The polarization curves}

\subsubsection{Model validation}

We first validated the model against the experimental data reported in [39] to ensure that it adequately represents the key electrochemical processes in the CL. In the validation, the thickness of the CL was 50 $\mu$ m taken from [38], and the GDL was assumed to be anisotropic and the effective binary coefficients of all gases in its in-plane and through-plane directions were described by

$$
\begin{aligned}
& D_{i j-\text { in }}^{e f f}=D_{i j} f_{\text {throuh }}(\varphi), \\
& D_{i j-\text { throuh }}^{\text {eff }}=D_{i j} f_{\text {throuh }}(\varphi),
\end{aligned}
$$

respectively, where $D_{i j}$ is the binary diffusion coefficient of gases $i$ and $j$ in free space, $\varphi$ is

GDL porosity and $f_{\text {in }}(\varphi)$ and $f_{\text {throuh }}(\varphi)$ describe the reduction of the diffusion coefficients in the in-plane and through-plane directions, respectively, due to the impact of GDL structure. The protonic conductivity and diffusion coefficients of gases in the CL depend on ionomer content, but their values were not available. We hence treated them as fitting parameters in the simulations. We also considered oxygen dissolution, but treated the specific outer surface area of the ionomer film as a fitting parameter as its value is not available. Other parameters were taken from [17].

The cell voltage was calculated from

$$
E=E_{t h}-I R_{m}-N C O,
$$

where $E_{\text {th }}$ is the theoretical oxygen reduction potential, $R_{m}$ is the resistance of the membrane and NCO is the potential difference between the solid potential at the GDL-plate interface and the electrolyte potential at the CL-membrane interface. The hydrogen diffusion and 
reaction in the anode were assumed to be fast and their associated potential loss was negligible. The resistance of the membrane used in the literature varies $[38,40]$, and we took an average value, $R_{m}=0.3 \Omega \cdot \mathrm{cm}^{2}$, in the simulations. The comparison between the simulated results and the experimental data is showed in Fig. 9A. They agree reasonably well. The simulated results slightly overestimated the potential loss at high current density probably due to the underestimation of the oxygen dissolution. The fitting could be improved by changing other parameters, but we made no further efforts in this direction as the purpose of the validation is to demonstrate that the model is able to capture the main features of the polarization curve.

To further test the agglomerate model, we also compared the model with the experimental polarization curve reported in [41]. The pressure of the gas mixture in the channel is 1atm, and the molar fraction of gaseous oxygen and the water vapour in the channel is 0.145 and 0.312 , respectively. The thickness of the GDL and CL is $270 \mu \mathrm{m}$ and $55 \mu \mathrm{m}$, respectively. The width of the channel and shoulder is $1000 \mu \mathrm{m}$, and the operation temperature was $70^{\circ} \mathrm{C}$. The diffusion coefficients of the GDL and CL were the same as that used in the above example. Again, since the specific outer surface area of the ionomer film is not known, we treated it and the oxygen dissolution rate as fitting parameters. We were unable to fit the data using $R_{m}=0.3 \Omega \cdot \mathrm{cm}^{2}$, and hence treated the membrane resistance as a fitting parameter as well. The comparison between the best-fitting results and the experimental data is shown in Fig. 9B. Overall, they agree well. The simulation slightly overestimated the cell performance at high current density; this is likely due to the impact of liquid water which was neglected in our simulations.

\subsubsection{Impact of oxygen diffusion in ionomer film and agglomerate}

In the following analysis, we follow Sun et al. [17] and plot the average current density against the cathode potential by subtracting only NCO from $E_{t h}$. 
The ability of the agglomerate to conduct dissolved oxygen is represented by the parameter $\alpha$ in the agglomerate model, which, for a given CL, depends on operating temperature as shown in Fig.6B. To demonstrate the impact of operating temperature on catalyst efficiency, we simulated the cathode electrode at two temperatures. Their associated parameter $\alpha$ is $20000 \mathrm{~s}^{-1}$ and $55000 \mathrm{~s}^{-1}$ respectively. The ionomer film thickness was assumed to be $10 \mathrm{~nm}$, and the value of the parameter $\beta$ is 0.79 . Oxygen dissolution was assumed to be very fast and not a limiting factor. The simulated polarization curves are compared in Fig. 10. The figure shows that the impact of temperature is only noticeable when the cathode potential is less than $0.6 \mathrm{~V}$.

Ionomer film is another factor believed to affect cell performance, but the ionomer film thickness used in the literature varies widely, as thick as 80nm [17]. This appears to be an exaggeration as recent research revealed that the average ionomer film thickness is only 10nm [5]. We investigated the impact of ionomer film thickness by assuming that oxygen dissolution is fast and not a limiting factor. Other parameters used in the simulations were $\alpha=40000 \mathrm{~s}^{-1}$ and $\beta=0.79$. Fig. 11 compares the polarization curves when the agglomerate was coated by different ionomer films with their thickness ranging from 0 to $15 \mathrm{~nm}$. The figure reveals that the impact of the ionomer film is negligible when its thickness is less than $15 \mathrm{~nm}$.

\subsubsection{Impact of oxygen dissolution}

Gaseous oxygen needs to dissolve in the ionomer film first before it can move into the agglomerate to react with electron and proton on the catalyst sites. Most studies available in the literature assumed that oxygen dissolution is fast and not limiting factor, which leads to a debate about the importance of oxygen transport in the CL as recent tomography revealed that the average agglomerate size in modern CL is only about $100 \mathrm{~nm}$ rather than $1000 \mathrm{~nm}$ as in old CLs [42]. If the oxygen dissolution is fast, our simulations also supported that the 
potential loss due to oxygen transport in CL is not noticeable when the cathode potential is less than $0.7 \mathrm{~V}$. However, recent experiments also revealed that oxygen dissolution in real CL is not as fast as previously assumed, and it could become a limiting factor [36]. Based on the experimental results available in the literature, we estimated that the dissolution rate coefficient of oxygen is in the range of $k_{d}^{o}=0.0017 \mathrm{~ms}^{-1}$. We investigated the influence of oxygen dissolution on potential loss. In all simulations, the ionomer film was assumed to be15nm thick. Based on the image shown in Fig.2B, we estimated the specific outer surface area of the ionomer film, and the result is $9200000 \mathrm{~m}^{2} \mathrm{~m}^{-3}$. Other parameters used in the simulations are $\alpha=6000 \mathrm{~s}^{-1}, \beta=0.79$ and $k=1.35$. Fig.12 compares the polarization curves obtained when oxygen dissolution was assumed to be fast and not a limiting factor (achieved by assuming $k_{d}^{o}=10^{10} \mathrm{~ms}^{-1}$ ) with that when $k_{d}^{o}=0.00017 \mathrm{~ms}^{-1}$. It is evident that the potential loss induced by oxygen dissolution is enormous, consistent with the experimental results of Suzuki et al.[36]. This might explain why the ionomer film thickness used by the spherical models in the literature needs to be exaggerated to 80nm; this appears to compensate the resistance caused by oxygen dissolution which is ignored in the spherical models [17].

\section{Conclusions}

The sluggish oxygen reduction in the cathode CL of PEM fuel cell is affected by a number of processes, and understanding each process and linking it back to CL structure is critical in fuel cell modelling and CL design. Whilst the spherical agglomerate models have been the dominant model in the literature to describe CL in fuel cell modelling, they are just to introduce a resistance to oxygen transport and reaction rather than a geometrical description of the CL.

The application of tomography technology in fuel cell over the past decade is able to acquire 3D structures of the CL at resolutions of a few nanometres. This can be combined with pore-scale simulation to directly calculate the average electrochemical reaction rate. In 
numerical modelling, however, the CL needs to be divided into a number of grids, and the average reaction rate in each grid has to be calculated separately. Since the electrochemical reaction depends on overpotential which varies spatially within the CL, calculating the average reaction rate in each grid through pore-scale simulation is extremely time-consuming. It is hence more rationale to find a simple method that can calculate the average reaction rate with an accurate description of the CL structure and other agglomerate processes. This paper presented such a formula.

The formula was derived by modelling the dissolved oxygen in the agglomerate, including the ionomer films, as two independent random processes. The first one is the probability that a dissolved oxygen molecule, which dissolves in the ionomer film on agglomerate surface, enters and continues to remain in the agglomerate as time elapses. The second one is the probability of the molecule being consumed in a reduction reaction as time passes. The probability distribution function of the first one depends only on agglomerate geometry and its ability to conduct oxygen, and can be directly calculated from the CL structure. The probability of the second process was derived by assuming that the oxygen reduction is first-order kinetic.

Based on a CL acquired using FIB/SEM tomography, we calculated the distribution functions of the first process for the CL with its agglomerate coated by different ionomer films. It is found that the distribution functions calculated under all conditions can be fitted to a generalised gamma distribution function, from which we are able to derive a simple analytical agglomerate model to calculate the oxygen reduction rate.

After gaseous oxygen moves from the GDL into the inter-agglomerate pores within the CL, it needs to dissolve in the ionomer film first prior to reacting with proton and electron inside the agglomerate. Oxygen dissolution depends on a number of processes and could become a limiting factor to oxygen reduction. Most spherical agglomerate models ignore this 
limitation, and account for it instead by either exaggerating the agglomerate diameter (up to $6000 \mathrm{~nm}$ ) or ionomer film thickness (up to $80 \mathrm{~nm}$ ) or both. Such treatments introduce an increased resistance to oxygen diffusion, but their results cannot be applied to CL design as they are not a correct description of the CL structure. In this paper, we extended the improved agglomerate model to include oxygen dissolution.

The accuracy of the improved agglomerate model for predicting the oxygen reaction was tested against the electrochemical reaction rates directly calculated from pore-scale simulation of oxygen diffusion and reaction in the CL with its agglomerate surface coated by different ionomer films. The results show good agreements.

The improved agglomerate model considers the resistances due to both oxygen dissolution and its consequent diffusion in the ionomer film and the agglomerate. To elucidate the relative significance of each resistance, we modelled a cathode electrode. We also validated the model against experimental data. The results indicated that for modern CLs with average agglomerate size of 100nm, the resistance caused by oxygen diffusion in the ionomer film and agglomerate is insignificant, and that most potential loss is caused by oxygen dissolution. Therefore, improving oxygen dissolution rate is a crucial way to improve fuel cell performance [43].This could have an important implication in CL design. It is understood that proton transport in CL is mainly through the ionomer film. An increase in ionomer film thickness can hence enhance protonic conductivity. On the other hand, since the inter-agglomerate pores are limited, an increase in ionomer content will reduce space for oxygen to diffuse and the contact areas between gaseous oxygen and ionomer film interface for oxygen to dissolve. This, when oxygen dissolution is the main limiting factor, could substantially reduce catalyst efficiency. Therefore, the ionomer content in a CL needs to be optimised [44]. In using numerical model to optimize ionomer content, an accurate 
description of the CL structure and the effect of ionomer film on proton transport and oxygen diffusion is crucial. The agglomerate model presented in this paper provides such a method.

One significant advantage of the proposed model in comparison with the classical spherical models is that it does not simplify the CL structure and all model parameters can be independently estimated from the CL structure. Moreover, it can be used to simulate transient behaviour of PEM fuel cell [45], which all other models could not.

\section{Acknowledgements}

Part of this research was supported by the UK Technology Strategy Board (TSB Project No. TP/6/S/K3032H).

\section{Appendix A}

If the Laplace transform of the function $g(t)$ is $G(s)$, the Laplace transforms of $g(t) \mathrm{e}^{\left(-k_{c} t\right)}$ and $\int_{0}^{t} g(\tau) d \tau$ are $G\left(s+k_{c}\right)$ and $G(s) / s$, respectively.

With the help of these relations, applying the Laplace transform to Eq.(11) with $C_{0}(t)$ being a constant gives

$$
\begin{aligned}
\bar{r}(s) & =k_{c} C_{0} \frac{1}{s} G\left(s+k_{c}\right) \\
& =k_{c} C_{0} \frac{1}{s}\left[1+\left(\frac{s+k_{c}}{\alpha}\right)^{\beta}\right]^{k},
\end{aligned}
$$

where

$$
\begin{aligned}
& \bar{r}(s)=\int_{0}^{\infty} R_{e}(t) \exp (-s t) d t, \\
& G(s)=\int_{0}^{\infty} g(t) \exp (-s t) d t .
\end{aligned}
$$

In the limit,

$$
R_{e}=r(t)=\bar{r}(s)=R_{e o}
$$

From Eq. (A1) we have 
$\bar{r}(s)=k_{c} C_{0} \frac{1}{s}\left[\frac{1}{1+\left(k_{c} / \alpha\right)^{\beta}}\right]^{k}$.

Applying the inverse-Laplace transform to (A4) gives the generalized agglomerate model:

$R_{e o}=r(t)=L^{-1}[\bar{r} \underset{s \rightarrow \infty}{\bar{r}}(s)]=k_{c} C_{0}\left[1+\left(k_{c} / \alpha\right)^{\beta}\right]^{-k}$,

where $L^{-1}[$ ]means taking inverse-Laplace transform. 


\section{References}

[1] X.M. Zhang, X.X. Zhang, Fuel Cells, 14 (2014) 303-311.

[2] N. Khajeh-Hosseini-Dalasm, M. Fesanghary, K. Fushinobu, K. Okazaki, Electrochim. Acta, 60 (2012) 55-65.

[3] M. Secanell, K. Karan, A. Suleman, N. Djilali, Electrochim. Acta, 52 (2007) 6318-6337.

[4] S. Thiele, T. Furstenhaupt, D. Banham, T. Hutzenlaub, V. Birss, C. Ziegler, R. Zengerle, Journal of Power Sources, 228 (2013) 185-192.

[5] E. Sadeghi, A. Putz, M. Eikerling, J. Solid State Electrochem., 18 (2014) 1271-1279.

[6] Y. Xiao, J.L. Yuan, B. Sunden, J. Electrochem. Soc., 159 (2012) B251-B258.

[7] Q.P. Wang, M. Eikerling, D.T. Song, Z.S. Liu, J. Electroanal. Chem., 573 (2004) 61-69.

[8] N.A. Siddique, F.Q. Liu, Electrochim. Acta, 55 (2010) 5357-5366.

[9] L. Xing, M. Mamlouk, R. Kumar, K. Scott, Int. J. Hydrog. Energy, 39 (2014) 9087-9104.

[10] M. Chisaka, H. Daiguji, Electrochem. Commun., 8 (2006) 1304-1308.

[11] T. Hutzenlaub, J. Becker, R. Zengerle, S. Thiele, Journal of Power Sources, 227 (2013)

260-266.

[12] S. Kamarajugadda, S. Mazumder, Journal of Power Sources, 183 (2008) 629-642.

[13] D. Harvey, J.G. Pharoah, K. Karan, Journal of Power Sources, 179 (2008) 209-219.

[14] S. Kamarajugadda, S. Mazumder, Journal of Power Sources, 208 (2012) 328-339.

[15] P.K. Das, X.G. Li, Z.S. Liu, Journal of Power Sources, 179 (2008) 186-199.

[16] E.W. Thiele, Industrial and Engineering Chemistry, 31 (1939) 916-920.

[17] W. Sun, B.A. Peppley, K. Karan, Electrochim. Acta, 50 (2005) 3359-3374.

[18] W.K. Epting, J. Gelb, S. Litster, Adv. Funct. Mater., 22 (2012) 555-560.

[19] S. Thiele, R. Zengerle, C. Ziegler, Nano Research, 4 (2011) 849-860.

[20] C. Ziegler, S. Thiele, R. Zengerle, Journal of Power Sources, 196 (2011) 2094-2097.

[21] R. Singh, A.R. Akhgar, P.C. Sui, K.J. Lange, N. Djilali, J. Electrochem. Soc., 161 (2014)

F415-F424.

[22] A.A. Shah, G.S. Kim, W. Gervais, A. Young, K. Promislow, J. Li, S. Ye, Journal of Power Sources, 160 (2006) 1251-1268.

[23] N.P. Siegel, M.W. Ellis, D.J. Nelson, M.R. von Spakovsky, Journal of Power Sources, 115 (2003) 81-89.

[24] A. Abedini, B. Dabir, M. Kalbasi, Int. J. Hydrog. Energy, 37 (2012) 8439-8450.

[25] R. Roshandel, F. Ahmadi, Renew. Energy, 50 (2013) 921-931.

[26] S. Obut, E. Alper, Journal of Power Sources, 196 (2011) 1920-1931.

[27] L. Xing, M. Mamlouk, K. Scott, Energy, 61 (2013) 196-210.

[28] F.C. Cetinbas, S.G. Advani, A.K. Prasad, Journal of Power Sources, 250 (2014) 110-119.

[29] S. Zils, M. Timpel, T. Arlt, A. Wolz, I. Manke, C. Roth, Fuel Cells, 10 (2010) 966-972.

[30] W.K. Epting, S. Litster, Int. J. Hydrog. Energy, 37 (2012) 8505-8511.

[31] X.X. Zhang, H. Ostadi, K.L. Jiang, R. Chen, Electrochim. Acta, 133 (2014) 475-483.

[32] X.X. Zhang, Y. Gao, H. Ostadi, K.L. Jiang, R. Chen, Electrochim. Acta, 150 (2014) 320-328.

[33] L. Xing, P.K. Das, X.G. Song, M. Mamlouk, K. Scott, Appl. Energy, 138 (2015) 242257.

[34] H.A. Vandervorst, Siam Journal on Scientific and Statistical Computing, 13 (1992) 631644.

[35] V. Cvetkovic, Water Resources Research, 48 (2012) 12.

[36] T. Suzuki, K. Kudo, Y. Morimoto, Journal of Power Sources, 222 (2013) 379-389.

[37] X. Zhang, Y. Gao, H. Ostadi, K. Jiang, R. Chen, Int. J. Hydrog. Energy, 39 (2014) 17222-17230.

[38] M. Moein-Jahromi, M.J. Kermani, Int. J. Hydrog. Energy, 37 (2012) 17954-17966. 
[39] E.A. Ticianelli, C.R. Derouin, A. Redondo, S. Srinivasan, J. Electrochem. Soc., 135 (1988) 2209-2214.

[40] J.J. Zhang, W. Yang, L. Xu, Y.X. Wang, Electrochim. Acta, 56 (2011) 6912-6918.

[41] C.Y. Jung, C.H. Park, Y.M. Lee, W.J. Kim, S.C. Yi, Int. J. Hydrog. Energy, 35 (2010) 8433-8445.

[42] A.A. Kulikovsky, Electrochim. Acta, 130 (2014) 826-829.

[43] T. Kim, S.D. Yim, Y.W. Choi, T.H. Yang, Y.G. Yoon, S.H. Park, C.S. Kim, I.K. Sung, Electrochem. Commun., 13 (2011) 1313-1316.

[44] J.H. Jung, M.S. Cha, J.B. Kim, J. Nanosci. Nanotechnol., 12 (2012) 5412-5417.

[45] S.M. Chang, H.S. Chu, Journal of Power Sources, 161 (2006) 1161-1168. 
Table 1 Physical properties and other parameters used in the simulations

\begin{tabular}{|c|c|}
\hline Parameters & Value \\
\hline Cell temperature (K) & 323.15 \\
\hline Fraction of ionomer in agglomerate pores (\%) & 30 \\
\hline Oxygen diffusion coefficient in ionomer $\left(\mathrm{m}^{2} \mathrm{~s}^{-1}\right)$ & $8.45 \times 10^{-10}$ \\
\hline Oxygen reference concentration $\left(\mathrm{mol} \mathrm{m}^{-3}\right)$ & 0.85 \\
\hline Cathode transfer coefficient & $\begin{array}{ll}1.0 & \eta \geq 0.8 \mathrm{~V} \\
0.61 & \eta<0.8 \mathrm{~V}\end{array}$ \\
\hline Reference current exchange density $\left(\mathrm{mAm}^{-2}\right)$ & $\begin{array}{ll}0.385 & \eta \geq 0.8 \mathrm{~V} \\
15 & \eta<0.8 \mathrm{~V}\end{array}$ \\
\hline Active surface area of catalyst $\left(\mathrm{m}^{2} \mathrm{~m}^{-3}\right)$ & $2.4 \times 10^{7}$ \\
\hline Faraday constant $\left(\mathrm{C} \mathrm{mol}^{-1}\right)$ & 96485 \\
\hline Gas constant $\left(\mathrm{J} \mathrm{mol}^{-1} \mathrm{~K}^{-1}\right)$ & 8.314 \\
\hline Average protonic conductivity $\left(\mathrm{Sm}^{-1}\right)$ & 0.83 \\
\hline Average electronic conductivity of $\mathrm{GDL} \mathrm{Sm}^{-1}$ ) & 170 \\
\hline Average electronic conductivity of $\mathrm{CL}\left(\mathrm{Sm}^{-1}\right)$ & 50 \\
\hline Tortuosity of GDL in in-plane direction & 0.4 \\
\hline Tortuosity of GDL in through-plane direction & 0.3 \\
\hline Oxygen dissolution rate coefficient $\left(\mathrm{ms}^{-1}\right)$ & 0.00017 \\
\hline Tortuosity of the CL & 0.02 \\
\hline Inter-agglomerate porosity & 0.45 \\
\hline Henry constant $\left(\mathrm{atm} \mathrm{m} \mathrm{mol}^{-1}\right)$ & 0.3125 \\
\hline Resistance of the membrane $\left(\Omega \mathrm{cm}^{-2}\right)$ & 0.3 \\
\hline Theoretical oxygen reduction potential (V) & 1.229 \\
\hline Thickness of CL $(\mu \mathrm{m})$ & 20 \\
\hline Thickness of the GDL $(\mu \mathrm{m})$ & 250 \\
\hline Width of channel $(\mu \mathrm{m})$ & 1200 \\
\hline Width of land $(\mu \mathrm{m})$ & 1200 \\
\hline Binary diffusion of water vapour and oxygen $\left(\mathrm{m}^{2} \mathrm{~s}^{-1}\right)$ & $3.7 \times 10^{-5}$ \\
\hline Binary diffusion of oxygen and nitrogen $\left(\mathrm{m}^{2} \mathrm{~s}^{-1}\right)$ & $2.79 \times 10^{-5}$ \\
\hline Binary diffusion of nitrogen and water vapour $\left(\mathrm{m}^{2} \mathrm{~s}^{-1}\right)$ & $3.87 \times 10^{-5}$ \\
\hline $\begin{array}{l}\text { Effective diffusion coefficient of gaseous oxygen in } \\
\text { inter-agglomerate pores }\left(\mathrm{m}^{2} \mathrm{~s}^{-1}\right)\end{array}$ & $3.4 \times 10^{-6}$ \\
\hline $\begin{array}{l}\text { Effective diffusion coefficient of dissolved oxygen in } \\
\text { intra-agglomerate pores }\left(\mathrm{m}^{2} \mathrm{~s}^{-1}\right)\end{array}$ & $1.38 \times 10^{-10}$ \\
\hline
\end{tabular}




\section{Figure captions}

Fig. 1. Schematic illustration of the cathode electrode and a numerical CL grid created in numerical modelling, in which the gaseous oxygen moves into the inter-agglomerate pores first and then dissolves in the ionomer film. The detailed oxygen diffusion and reaction in the agglomerates within the grid cannot be explicitly resolved in the fuel cell modelling; instead, they are approximated by an agglomerate model using the volumetric average oxygen concentration and protonic and electronic potentials in the grid.

Fig. 2. The CL structure acquired using focused ion beam/scanning electron microscopy at resolution of 5 nanometres (A). The simulated CL with its agglomerates (green) coated numerically by ionomer films (red) (B); the inter-agglomerate pores are represented by blue.

Fig. 3. Cross sections of the simulated CL with its agglomerates (green) coated numerically by ionomer films (red) with different thicknesses; the inter-agglomerate pores are represented by blue. (A) Without ionomer, (B) coated by an ionomer film with dimensionless thickness $\lambda^{\prime}=1$, (B) coated by an ionomer film with dimensionless thickness $\lambda^{\prime}=2$, (D) coated by an ionomer film with dimensionless thickness $\lambda^{\prime}=3$.

Fig. 4. The impact of ionomer-film thickness on the probability distribution function $g\left(t^{\prime}\right)$ calculated from simulating the movement of dissolved oxygen in the agglomerate within the CL shown in Fig. 2B, and the ionomer films shown in Fig.3.

Fig. 5. Comparison between the Laplace-transformed distribution functions $g\left(t^{\prime}\right)$ calculated from simulating movement of the dissolved oxygen (solid lines) with that fitted by the proposed model (broken lines) for the agglomerates shown in Fig.2B coated by different ionomer films as shown in Fig.3.

Fig. 6. Change of the parameter $k$ in the agglomerate model with the normalised ionomerfilm thickness $\lambda^{\prime}$ (A). Change of the parameter $\alpha\left(s^{-1}\right)$ in the model with temperature (B).

Fig. 7. Comparison between the effectiveness factors directly calculated from the pore-scale simulations of oxygen diffusion and reaction (symbols) and that predicted by the model (solid lines) with its parameters estimated from curve-fitting as shown in Fig. 5.

Fig. 8. Comparison between the effectiveness factors directly calculated from pore-scale simulations (broken lines) with that predicted from the agglomerate model (solid lines) when oxygen dissolution becomes a limiting factor.

Fig. 9. Comparison between experimental polarization curves and the results simulated using the proposed model. (A) Comparison with the experimental data reported in Ticianelli et al [39]. (B) Comparison with the experimental data reported in Jung et al [41]. 
Fig. 10. Impact of the ability of agglomerate to conduct oxygen (represented by the parameter $\alpha)$ on the polarization curves; the ionomer was assumed to be $15 \mathrm{~nm}$ thick $(k=1.35)$ and the parameter $\beta$ is 0.79 .

Fig. 11. Impact of the ionomer film thickness on polarization curves. Other parameters used in the simulations to obtain these curves are $\alpha=400000 \mathrm{~s}^{-1}$ and $\beta=0.79$.

Fig.12. Impact of oxygen dissolution in the ionomer film on polarization curves. The ionomer film thickness is $10 \mathrm{~nm}$, and other parameters used in the simulations to obtain the curves are $\alpha=600000 \mathrm{~s}^{-1}$ and $\beta=0.79$.

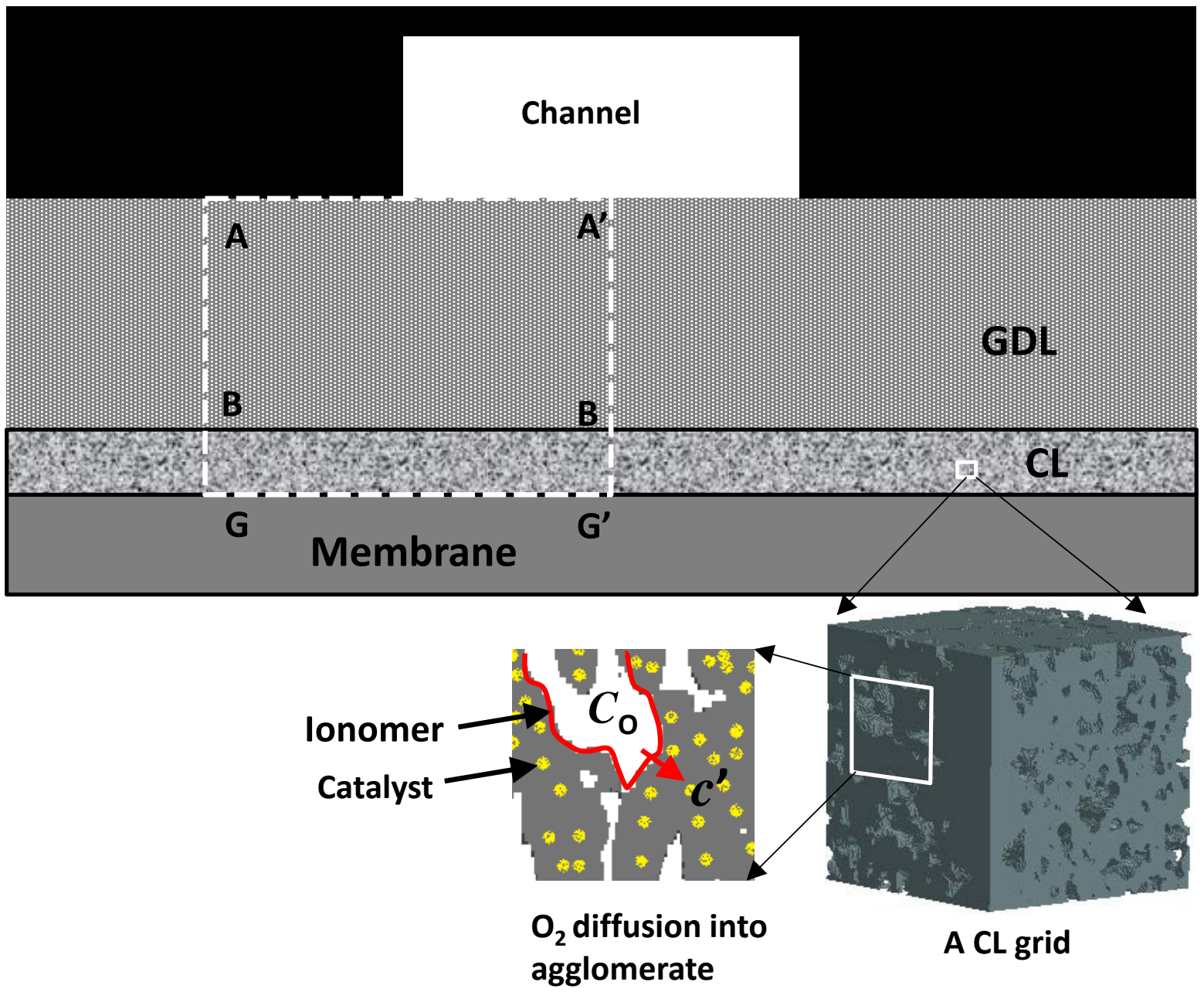

Fig. 1. Schematic illustration of the cathode electrode and a numerical CL grid created in numerical modelling, in which the gaseous oxygen moves into the inter-agglomerate pores first and then dissolves in the ionomer film. The detailed oxygen diffusion and reaction in the agglomerates within the grid cannot be explicitly resolved in the fuel cell modelling; instead, 
they are approximated by an agglomerate model using the volumetric average oxygen concentration and protonic and electronic potentials in the grid. 


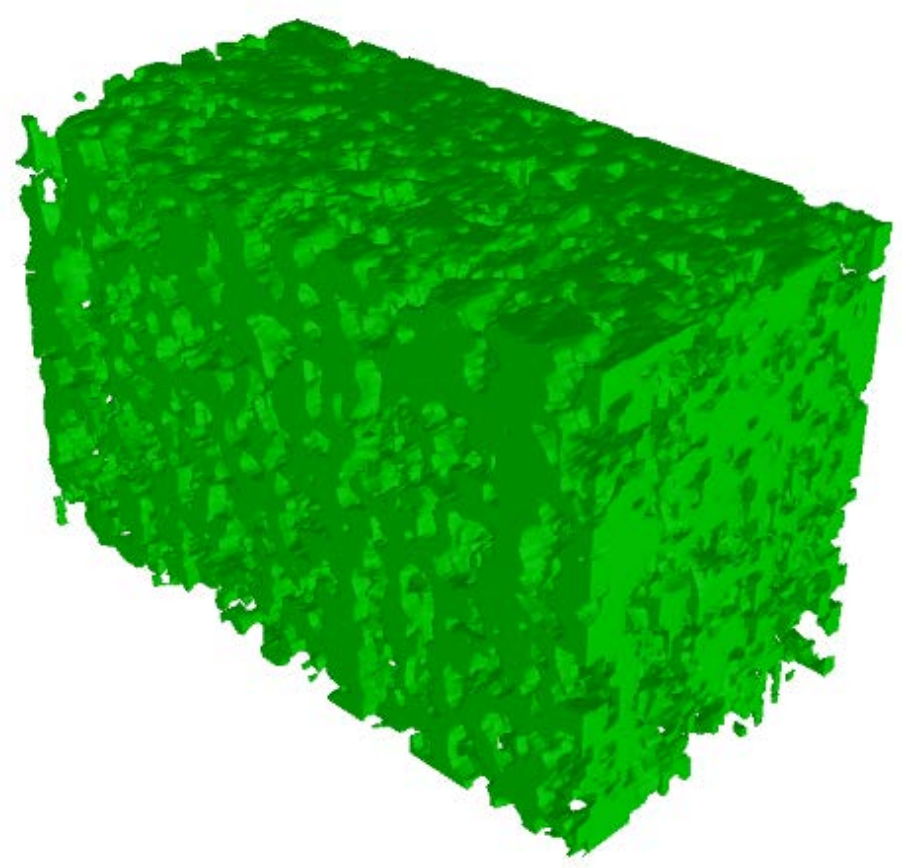

(A)

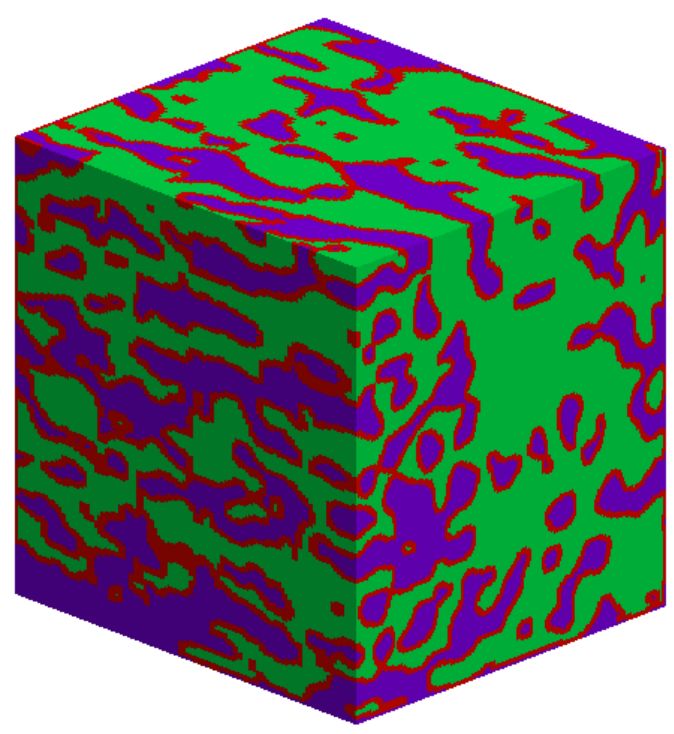

(B)

Fig. 2. The CL structure acquired using focused ion beam/scanning electron microscopy at resolution of 5 nanometres (A). The simulated CL with its agglomerates (green) coated numerically by ionomer films (red) (B); the inter-agglomerate pores are represented by blue. 
$\lambda^{\prime}=0(\mathrm{~A})$

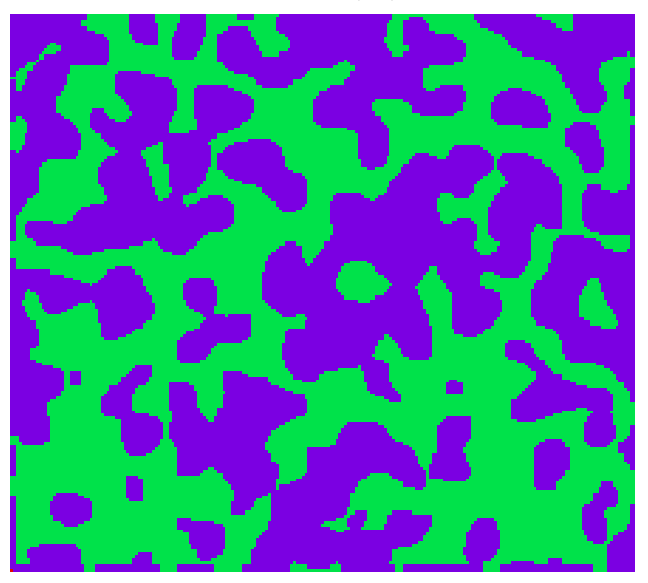

$\lambda^{\prime}=2(\mathrm{C})$

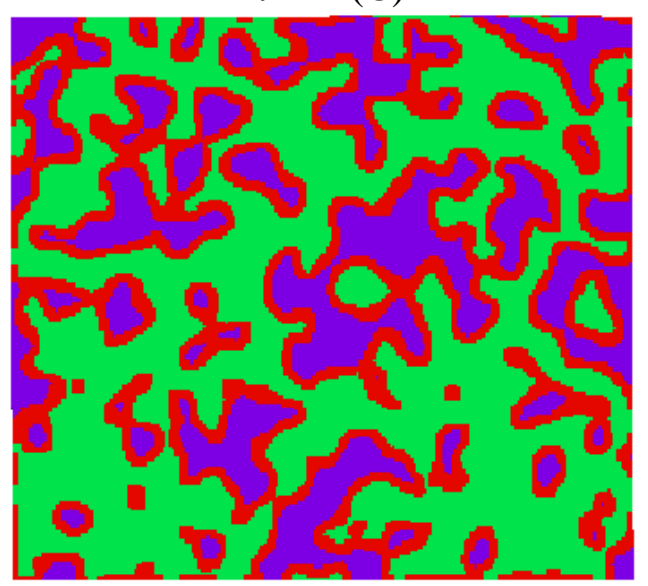

$\lambda^{\prime}=1(\mathrm{~B})$

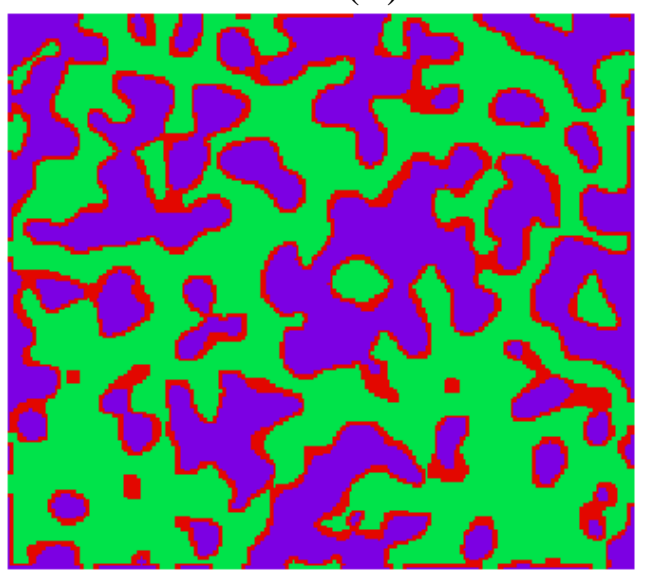

$\lambda^{\prime}=3(\mathrm{D})$

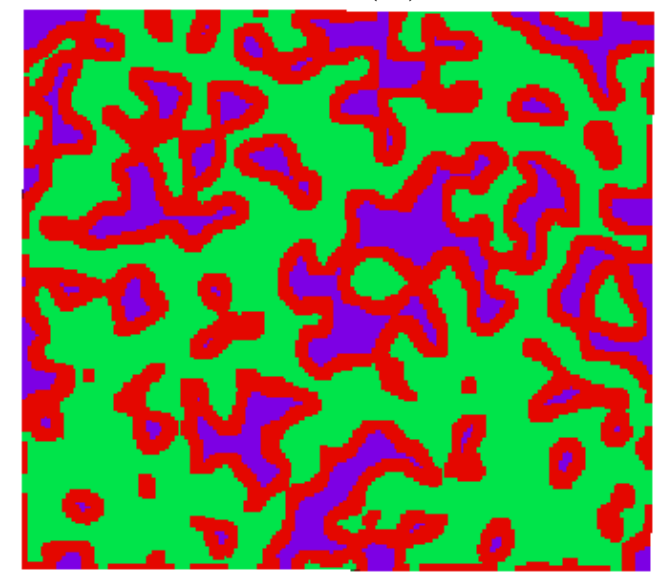

Fig. 3. Cross sections of the simulated catalyst layer with its agglomerates coated numerically by ionomer films of different thicknesses. (A) Without ionomer, (B) by an ionomer film with dimensionless thickness $\lambda^{\prime}=1$, (B) an ionomer film with dimensionless thickness $\lambda^{\prime}=2$, (D) ionomer film with dimensionless thickness $\lambda^{\prime}=3$. 


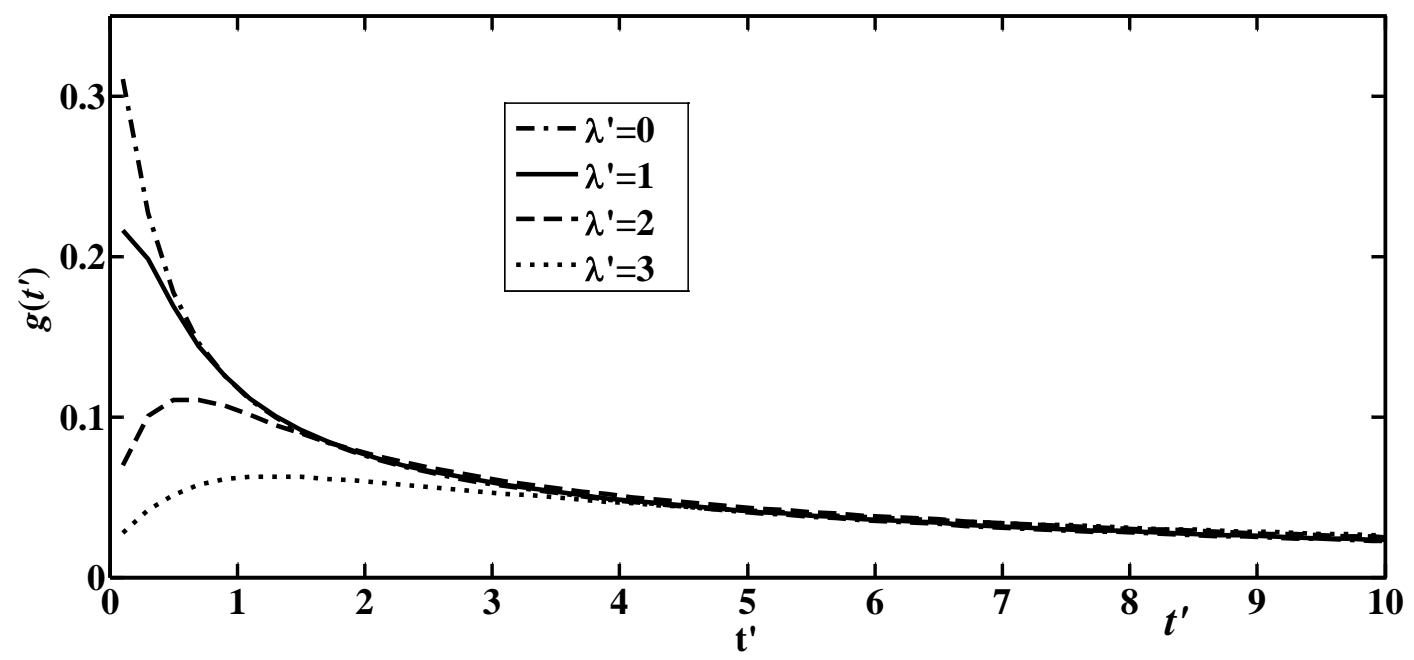

Fig. 4. The impact of ionomer-film thickness on the probability distribution function $g(t)$ calculated from simulating the movement of dissolved oxygen molecules in the agglomerate within the catalyst layer shown in Fig. 2B, and ionomer films shown in Fig.3. 

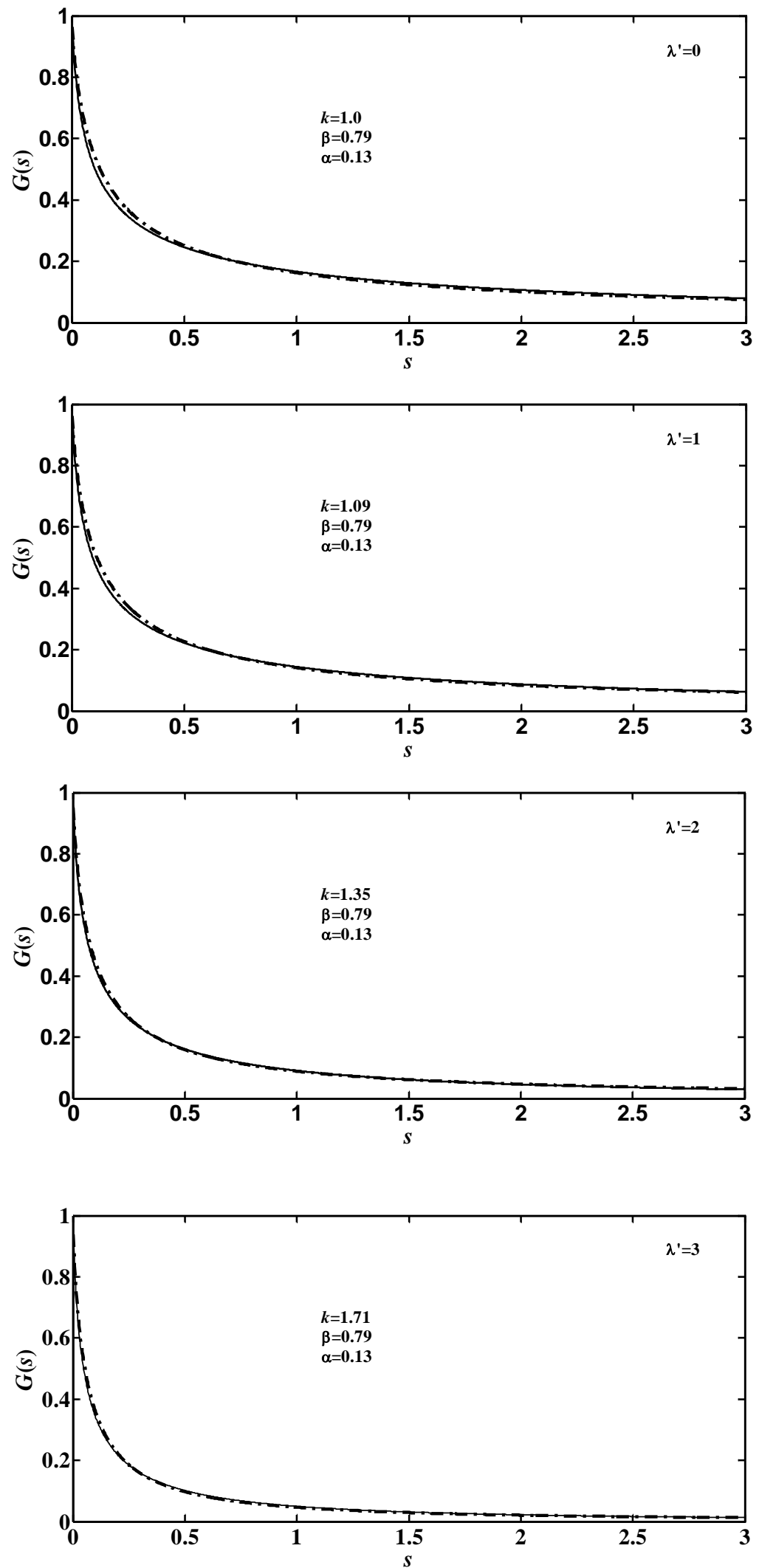

Fig. 5. Comparison between the Laplace-transformed distribution functions $g(t)$ calculated from simulating movement of the dissolved molecules (solid lines) with that fitted by the proposed model (broken lines) for the agglomerates shown in Fig.2 coated by different ionomer films shown in Fig.3. 
(A)

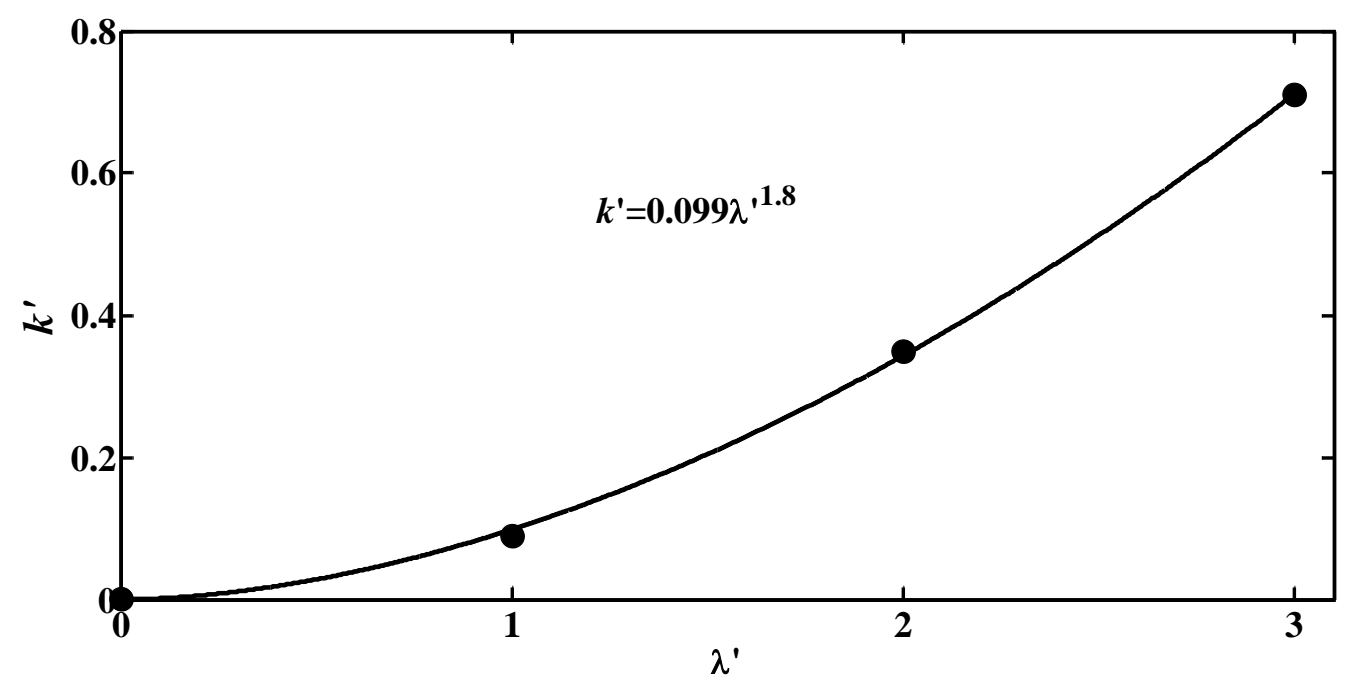

(B)

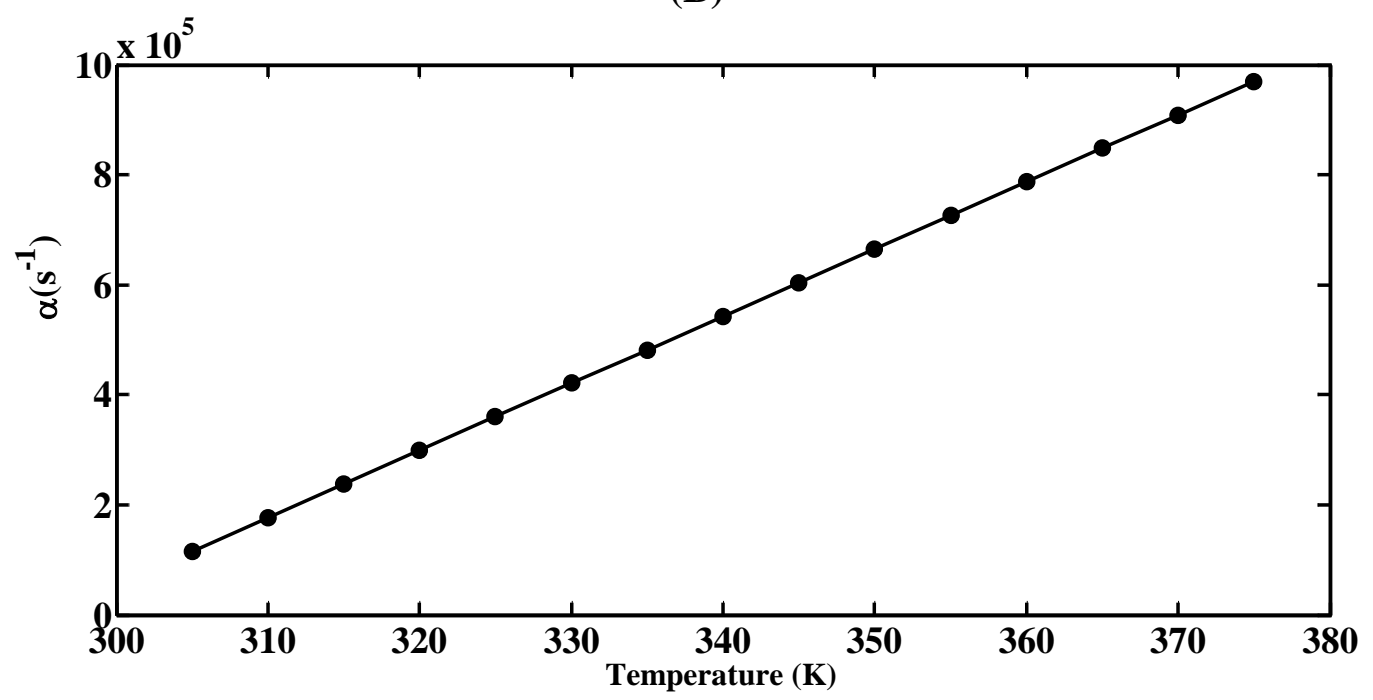

Fig. 6. (A) Change of the parameter $k$ in the agglomerate model with the normalised ionomer-film thickness $\lambda^{\prime}$. (B) Change of the parameter $\alpha\left(s^{-1}\right)$ in the model with temperature. 

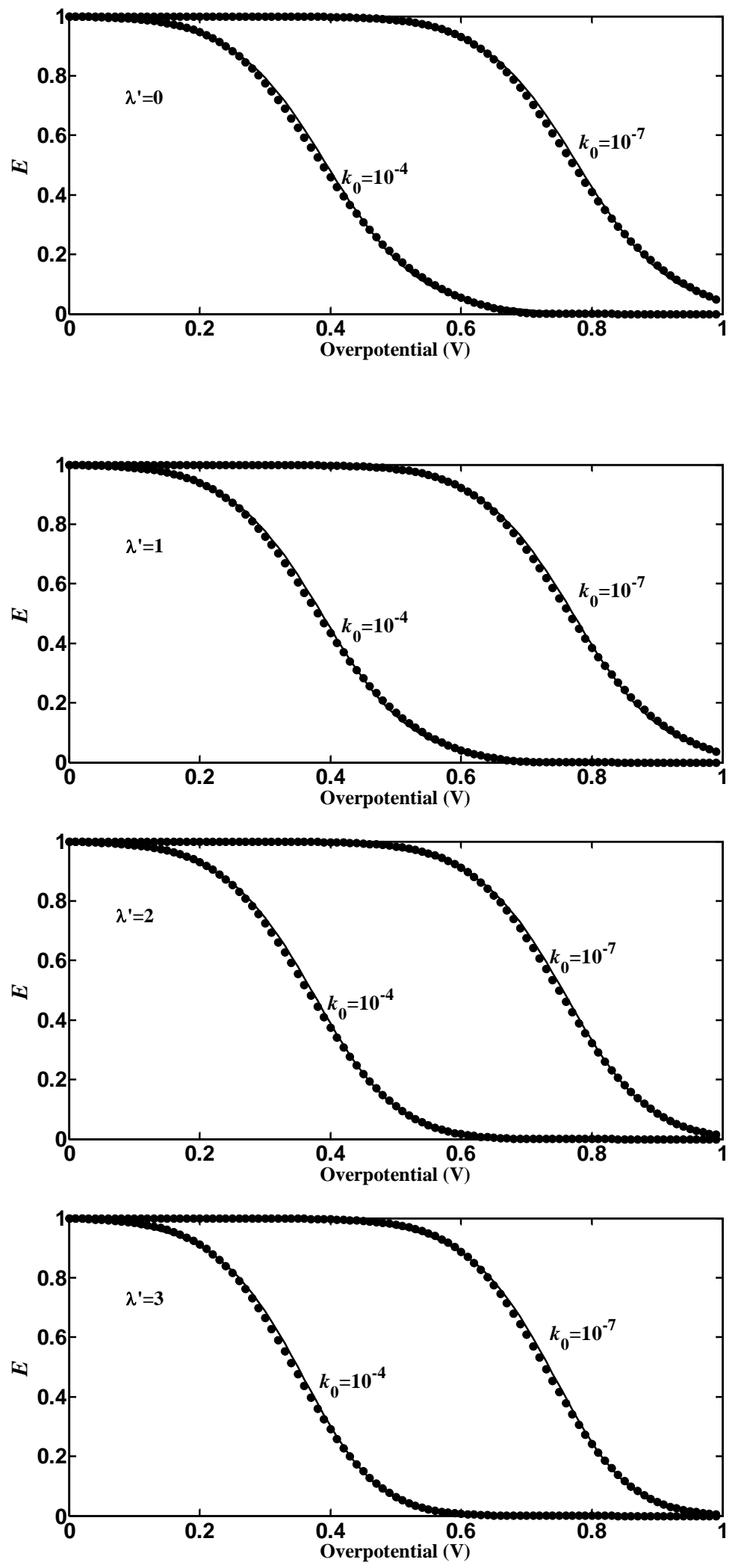

Fig. 7. Comparison between the effectiveness factors directly calculated from the pore-scale simulations of oxygen diffusion and reaction (symbols) and that predicted by the model (solid lines) with its parameters estimated from curve-fitting as shown in Fig. 5. 

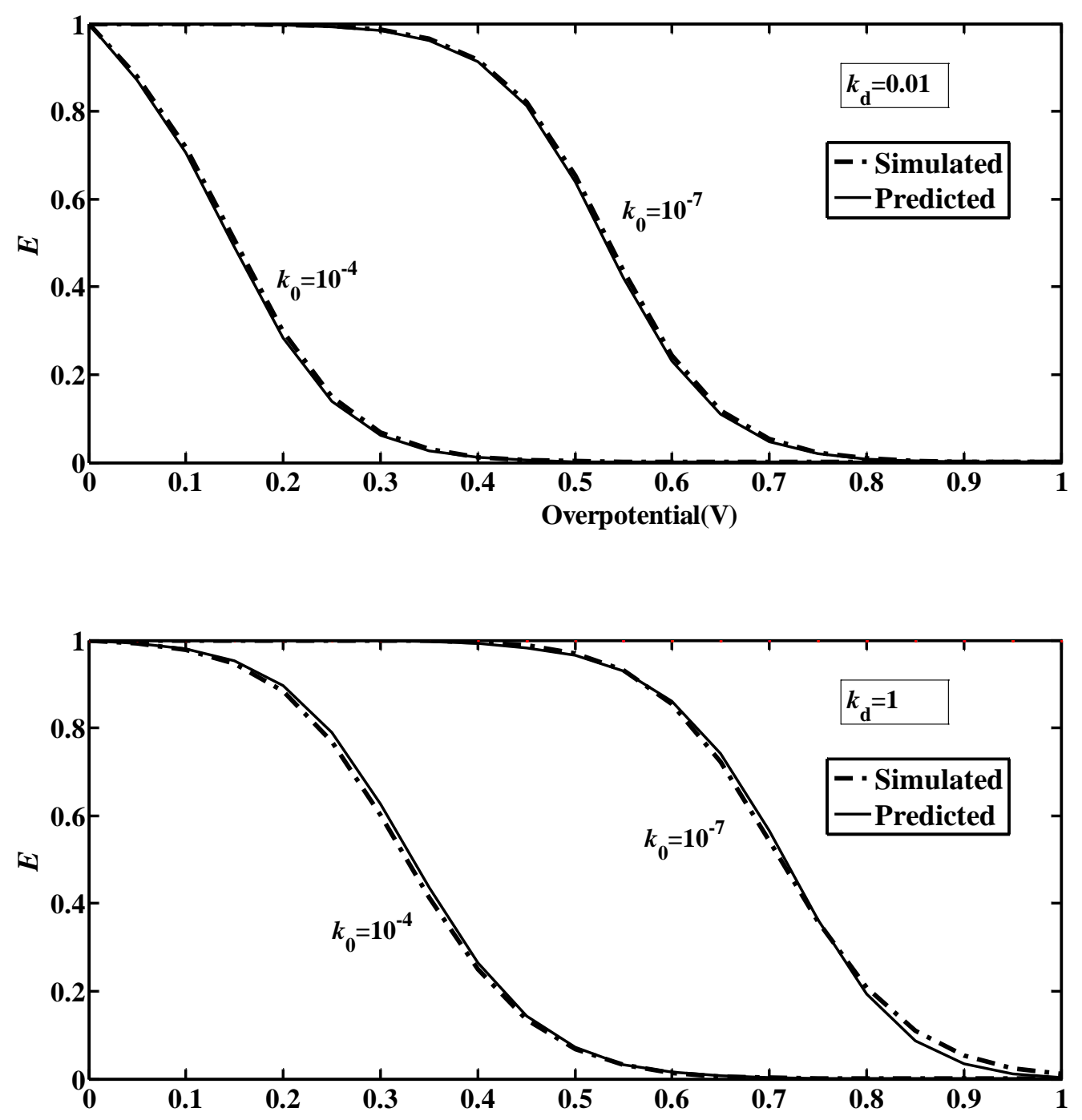

Fig. 8. Comparison between the effectiveness factors directly calculated from pore-scale simulations (broken lines) with that predicted from the agglomerate model (solid lines) when oxygen dissolution becomes a limiting factor. 


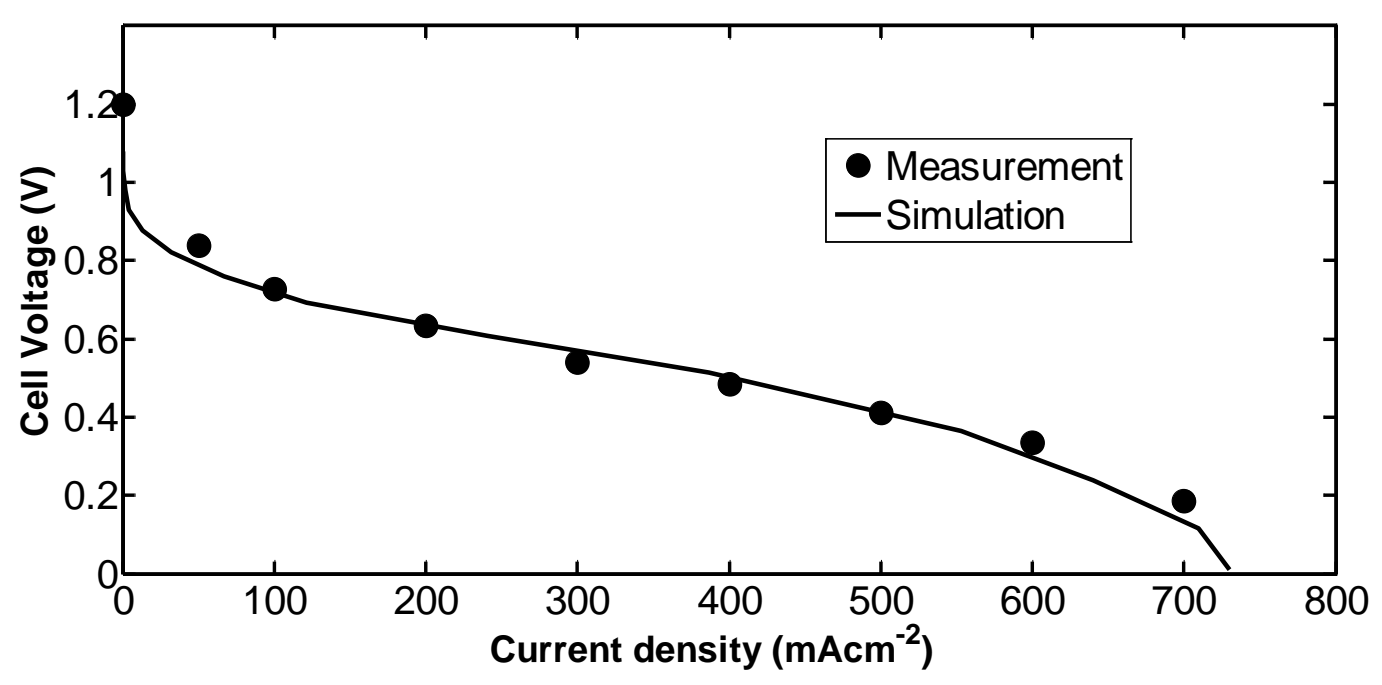

(B)

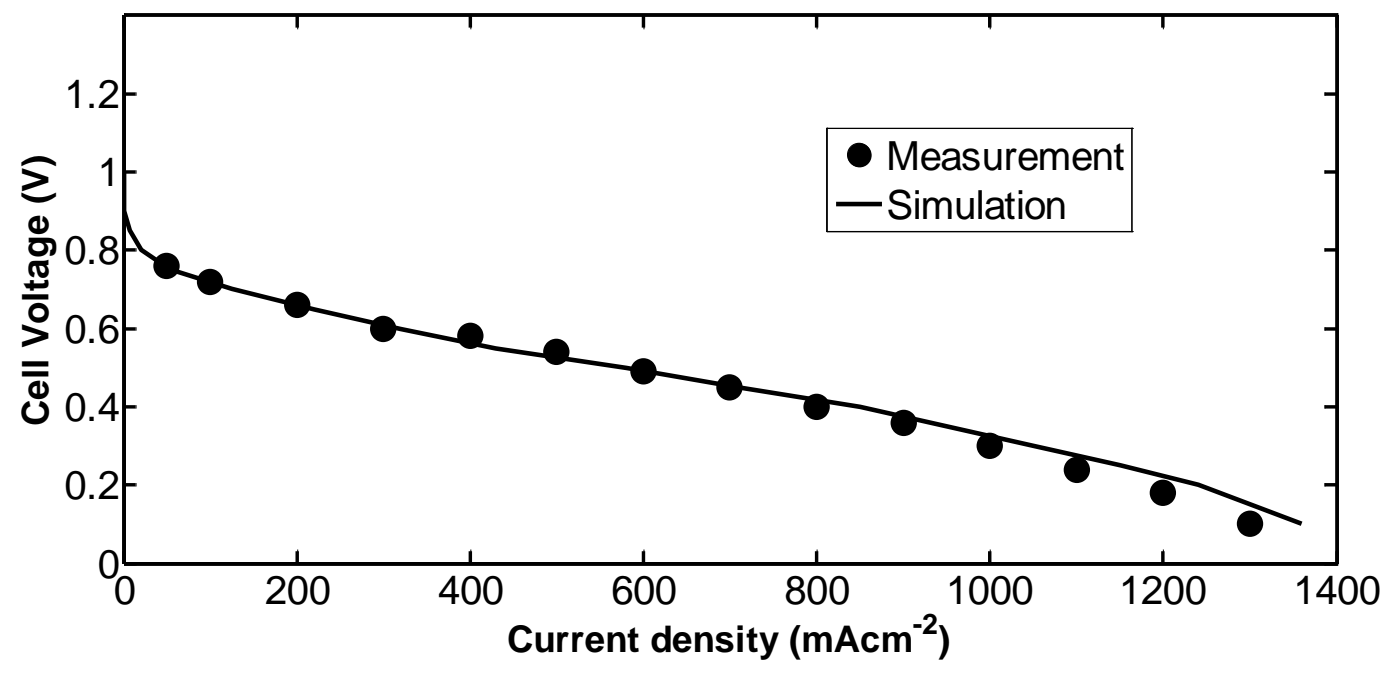

Fig. 9. Comparison between experimental polarization curves and the results simulated using the proposed model. (A) Comparison with the experimental data reported in Ticianelli et al [39]. (B) Comparison with the experimental data reported in Jung et al [41]. 


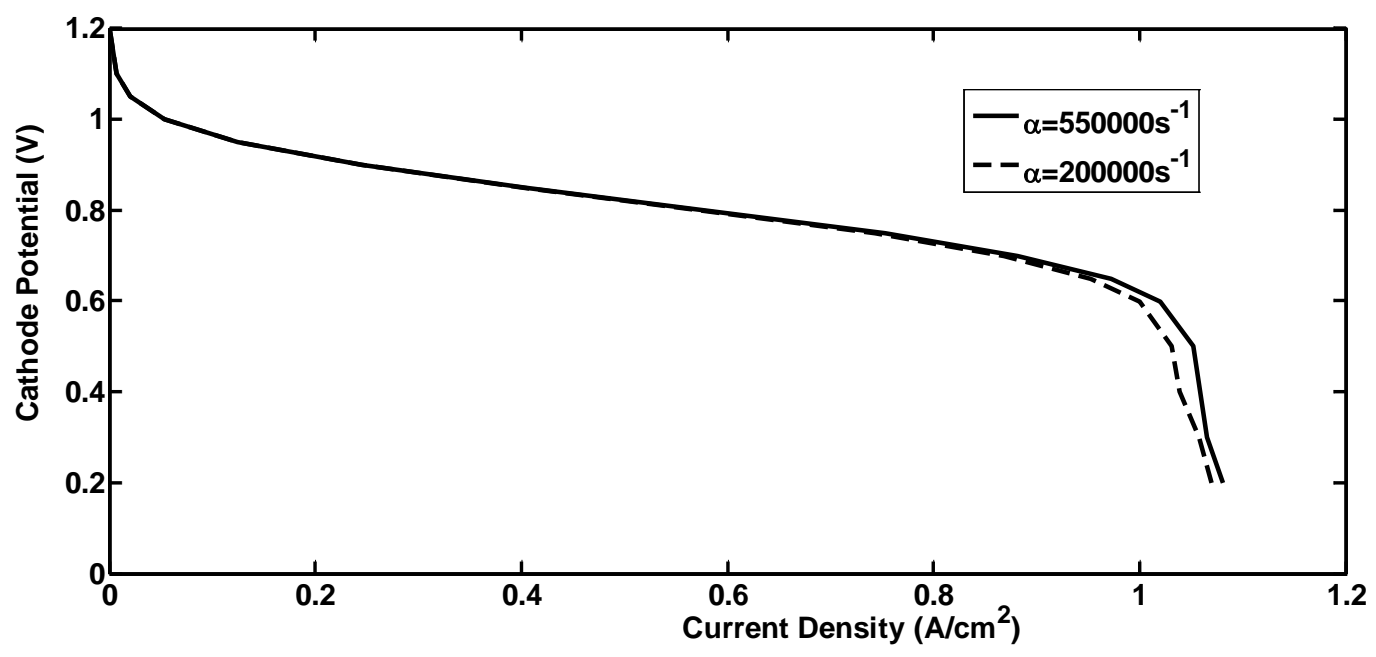

Fig. 10. Impact of the ability of agglomerate to conduct oxygen (represented by the parameter $\alpha)$ on the polarization curves; the ionomer was assumed to be $15 \mathrm{~nm}$ thick $(k=1.35)$ and the parameter $\beta$ is 0.79 . 


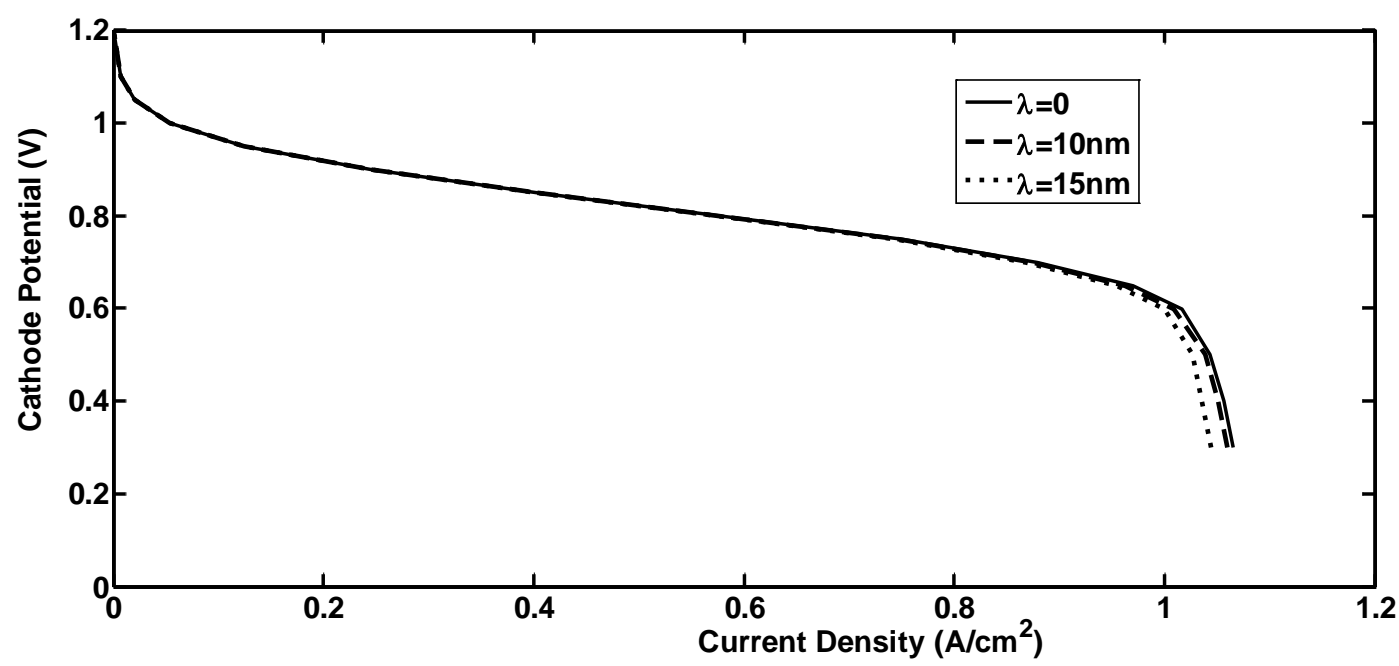

Fig. 11. Impact of ionomer film thickness on polarization curves. Other parameters used in the simulations to obtain these curves are $\alpha=400000 \mathrm{~s}^{-1}$ and $\beta=0.79$.

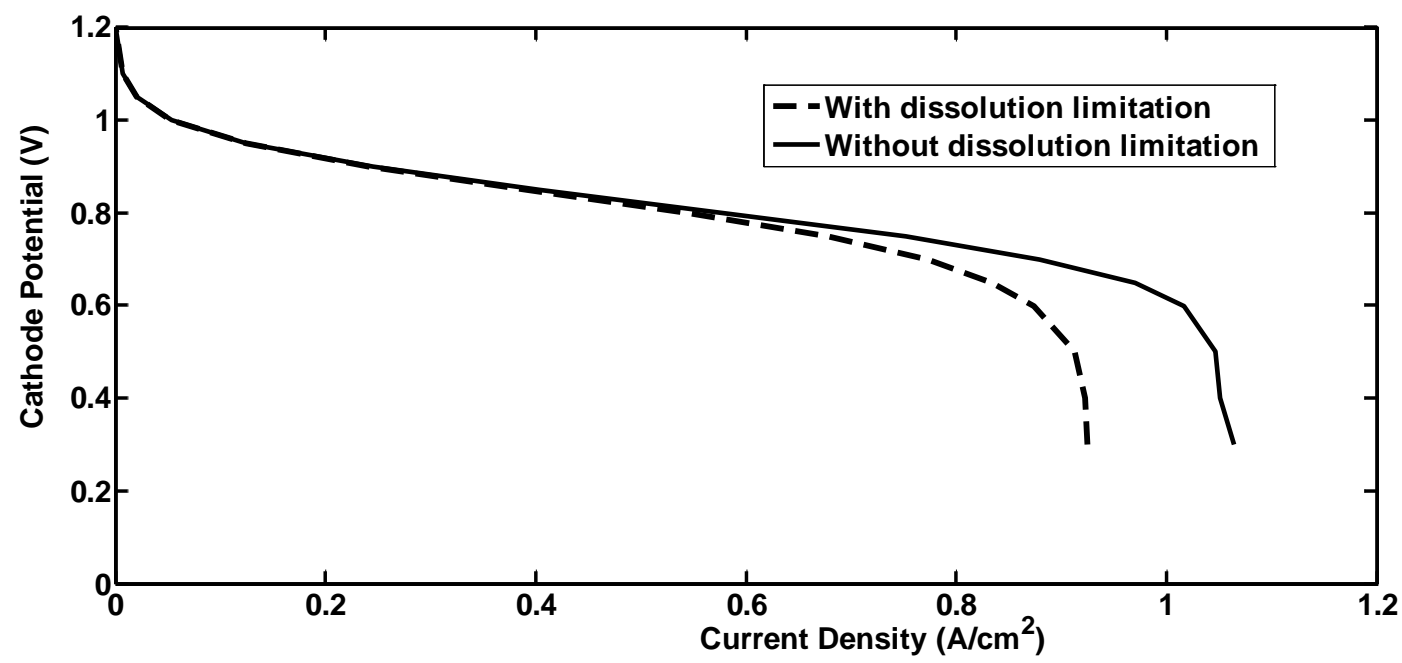

Fig.12. Impact of oxygen dissolution in the ionomer film on polarization curves. The ionomer film thickness is $10 \mathrm{~nm}$, and other parameters used in the simulations to obtain the curves are $\alpha=600000 \mathrm{~s}^{-1}$ and $\beta=0.79$. 